$$
S 4306
$$

\title{
TRIANGULATIONS OF CYCLIC POLYTOPES
} AND HIGHER BRUHAT ORDERS

by

JÖRG RAMBAU

No. 496/1996 



\title{
TRIANGULATIONS OF CYCLIC POLYTOPES AND HIGHER BRUHAT ORDERS
}

\author{
JÖRG RAMBAU
}

\begin{abstract}
Recently EDELMAN \& REINER suggested two poset structures $\mathcal{S}_{1}(n, d)$ and $\mathcal{S}_{2}(n, d)$ on the set of all triangulations of the cyclic $d$ polytope $C(n, d)$ with $n$ vertices. Both posets are generalizations of the well-studied Tamari lattice. While $\mathcal{S}_{2}(n, d)$ is bounded by definition, the same is not obvious for $S_{1}(n, d)$. In the paper by EDELMAN \& REINER the bounds of $\mathcal{S}_{2}(n, d)$ were also confirmed for $\mathcal{S}_{1}(n, d)$ whenever $d \leq 5$, leaving the general case as a conjecture.

In this paper their conjecture is answered in the affirmative for all $d$, using several new functorial constructions. Moreover, a structure theorem is presented, stating that the elements of $S_{1}(n, d+1)$ are in oneto-one correspondence to certain equivalence classes of maximal chains in $S_{1}(n, d)$. In order to clarify the connection between $S_{1}(n, d)$ and the higher Bruhat order $\mathcal{B}(n-2, d-1)$ of MANIN \& SCHECHTMAN, we define an order-preserving map from $\mathcal{B}(n-2, d-1)$ to $S_{1}(n, d)$, thereby concretizing a result by KAPRANOV \& VOEVODSKY in the theory of ordered $n$-categories.
\end{abstract}

\section{INTRODUCTION}

In this paper we examine the structure of the first higher Stasheff-Tamari order $S_{1}(n, d)$ on the set of all triangulations of the cyclic polytope $C(n, d)$ (definitions below), introduced by EDELMAN \& REINER [6]. It turns out that it is similarly structured as the higher Bruhat order $\mathcal{B}(n-2, d-1)$ of MANIN \& SCHECHTMAN [13]; in particular it is bounded.

Given a triangulation of the convex hull of a finite point configuration in Euclidean $d$-space that is not satisfying a certain quality measure, can one find a better, or even the best triangulation (with respect to this measure) by performing a finite sequence of (computational cheap) local transformations? A necessary condition for the latter case is that any possible triangulation is accessible by this kind of transformations. In particular, a repeatedly posed question in combinatorial and computational geometry (see for example BILlERA, KAPRANOV \& STURMFELS [3], EDELSBRUNNER [7, Open Problem 8], and JOE [10, Conjecture 1]) is whether or not any two triangulations of (the convex hull of) a given finite point configuration

The author is supported by the graduate school "Algorithmische Diskrete Mathematik" of the Deutsche Forschungsgemeinschaft (DFG), grant We 1265/2-1. 
in Euclidean space of dimension $d$ can be connected by a sequence of bistellar operations.

For $d=2$ the answer is affirmative, as is for the restriction to regular triangulations (by the work of GELFAND, KAPRANOV \& ZELEVINSKY [8]). For $d \geq 3$ and general triangulations, however, the problem is open in spite of many attacks in this direction.

Similar problems attained attention in several fields of pure mathematics, thereby leading to remarkable new concepts, such as the secondary polytope defined by GELFAND, KAPRANOV \& ZELEVINSKY [8]), further studied by Billera, Filliman \& StURMFels [1] and Billera, GelFAND \& STURMFELS [2]. The theoretical question behind this all is the following: Has the set of all triangulations of a point configuration a well-behaved global structure with respect to local transformations? A far-reaching generalization of this question to restricted polyhedral subdivisions was recently answered in the negative by RAMBAU \& ZIEGLER [14].

The cyclic $d$-polytope $C(n, d)$ with $n$ vertices appears on the scene as a combinatorially well-understood natural generalization of (convex) n-gons to higher dimensions. The triangulations of an $n$-gon form the extensively studied Tamari lattice - which one is definitely willing to consider as a good-natured structure in this context. (For a historical background on Tamari lattices and their different combinatorial interpretations we refer to the paper by EDELMAN \& REINER [6] and references given there.) The natural question now is which properties of the Tamari lattices survive in higher dimensions.

Since in general dimensions there are non-regular triangulations of cyclic polytopes (see BILlERA, GELFAND \& STURMFELS [2]) it is not a priori clear that the set of all triangulations of the cyclic polytope $C(n, d)$ is well-behaved. In the paper by EDELMAN \& REINER [6] two poset structures $S_{1}(n, d)$ and $S_{2}(n, d)$ are defined on this set, both generalizing the Tamari lattice and hence quite interesting from a purely combinatorial point of view. In the following we sketch their definitions.

The triangulations of the cyclic polytope $C(n, d)$ are in one-to-one correspondence to the piecewise linear sections from $C(n, d)$ into $C(n, d+1)$, according to the projection from $C(n, d+1)$ onto $C(n, d)$ that deletes the last coordinate. EDELMAN \& REINER [6] suggest two partial orders on all piecewise linear sections, and hence on the set of all triangulations of $C(n, d)$.

The first higher Stasheff-Tamari order $S_{1}(n, d)$ is defined by a covering relation between two sections if exactly one $(d+1)$-simplex fits between them in $C(n, d+1)$; the section that contains the upper facets of this simplex is defined to be greater than the other one. This corresponds to an increasing bistellar flip that replaces the lower facets of the $(d+1)$-simplex by the upper facets. Thus we get a purely combinatorial description of this poset in terms of local transformations. The second higher Stasheff-Tamari order 
$S_{2}(n, d)$ is defined geometrically via pointwise comparison of the heights of the sections.

While $\mathcal{S}_{2}(n, d)$ has a unique minimal element $\mathcal{F}^{l}(n, d+1)$ (the set of lower facets of $C(n, d+1)$ ) and a unique maximal element $\mathcal{F}^{u}(n, d+1)$ (the set of upper facets of $C(n, d+1)$ ), the same is not obvious for $S_{1}(n, d)$. On the other hand, the local structure of $S_{1}(n, d)$ is clear by definition while the covering relations in $S_{2}(n, d)$ are a priori unknown.

This motivated the following conjectures and results by EDELMAN \& REINER.

- For even $d$, both $\mathcal{S}_{1}(n, d)$ and $\mathcal{S}_{2}(n, d)$ are self-dual [6, Prop. 2.11, true in general].

- $S_{1}(n, d)$ coincides with $\mathcal{S}_{2}(n, d)[6$, Conj. 2.6, true for $d \leq 3]$.

- $\mathcal{F}^{l}(n, d+1)$ is the unique minimal element of $S_{1}(n, d)[6$, Conj. 2.7a, true for $d \leq 5$ ].

- $\mathcal{F}^{u}(n, d+1)$ is the unique maximal element of $S_{1}(n, d)[6$, Conj. 2.7b, true for $d \leq 4$ ].

- Any two triangulations of $C(n, d)$ are connected by a sequence of bistellar operations [6, Conj. 2.8, true for $d \leq 5]$.

- $\mathcal{S}_{1}(n, d)$ respectively $\mathcal{S}_{2}(n, d)$ is a lattice [6, Conj. 2.13, true for $\left.d \leq 3\right]$.

- In any interval of $S_{1}(n, d)$ respectively $S_{2}(n, d)$ distinct subsets of coatoms have different meets [6, Conj. 2.14, true for $d \leq 3]$.

Our main Theorem answers their Conjectures 2.7a, 2.7b, and 2.8 affirmatively and points out the connections between the triangulation posets in different dimensions. Its proof is completed in Section 5, using the functorial constructions in Section 3 which we consider as interesting in their own right.

\section{Theorem 1.1. (Main Result)}

(i) For all $n$ and all $d<n$ the first higher Stasheff-Tamari order $S_{1}(n, d)$ is bounded. The unique minimal element is the set $\mathcal{F}^{l}(n, d+1)$ of lower facets, the unique maximal element is the set $\mathcal{F}^{u}(n, d+1)$ of upper facets of $C(n, d+1)$.

(ii) The elements of $S_{1}(n, d+1)$ are in one-to-one-correspondence with the equivalence classes of maximal chains in $S_{1}(n, d)$ under the following equivalence relation: Two maximal chains are equivalent if they differ only by a permutation of their increasing bistellar operations.

(iii) Two maximal chains in $S_{1}(n, d)$ are equivalent if and only if they differ by a sequence of interchanges of consecutive bistellar operations that correspond to non-adjacent $(d+1)$-simplices in $C(n, d+1)$.

The following list of implications demonstrates the quantitative consequences of the main Theorem and the constructions provided in Section 3.

Corollary 1.2. For all $n$ and all $d<n$ the following hold: 
(i) The number of simplices in a triangulation of $C(n, d)$ lies between the number $\left(\begin{array}{c}n-\lceil d / 2\rceil-1 \\ d / 2\rfloor\end{array}\right)$ of upper facets and the number $\left(\begin{array}{c}n-\lceil(d+1) / 2\rceil \\ \lfloor(d+1) / 2\rfloor\end{array}\right)$ of lower facets of $C(n, d+1)$. In particular, for even $d$ all triangulations of $C(n, d)$ consist of $\left(\begin{array}{c}n-d / 2-1 \\ d / 2\end{array}\right)$ simplices.

(ii) The length of a maximal chain in $S_{1}(n, d)$ lies between the number $\left(\begin{array}{c}n-\lceil(d+1) / 2\rceil-1 \\ {[(d+1) / 2\rfloor}\end{array}\right)$ of upper facets and the number $\left(\begin{array}{c}n-\lceil d / 2\rceil-1 \\ \lfloor d / 2\rfloor+1\end{array}\right)$ of lower facets of $C(n, d+2)$. In particular, for odd $d$ the length of any maximal chain in $S_{1}(n, d)$ equals $\left(\begin{array}{c}n-(d+1) / 2-1 \\ (d+1) / 2\end{array}\right)$.

(iii) For even $d$ the diameter of the Hasse-diagram of $S_{1}(n, d)$ is between $\left(\begin{array}{c}n-d / 2-2 \\ d / 2\end{array}\right)$ and twice this value. For odd $d$ it is equal to $\left(\begin{array}{c}n-(d+1) / 2-1 \\ (d+1) / 2\end{array}\right)$.

(iv) For odd $d, S_{1}(n, d)$ is a ranked poset with rank function

$$
r(T):=\# \mathcal{F}^{l}(n, d+1)-\# T \quad \text { for all } T \in \mathcal{S}_{1}(n, d) .
$$

Theorem 1.1 points out a similarity to the structure of the higher Bruhat order $\mathcal{B}(n-2, d-1)$, a certain generalization of the weak Bruhat order on the symmetric group, defined by MANIN \& SCHECHTMAN [13] (see also ZIEGLER [15]). Previously, KAPRANOV \& VOEVODSKY [11] reported the existence of an order-preserving surjection from $\mathcal{B}(n-2, d-1)$ onto a poset structure on the set of all triangulations of $C(n, d)$ that is inherited by a certain ordered $n$-category. Unfortunately, it is not clear whether their poset structure is equivalent to $S_{1}(n, d)$. This led us to the investigations in Section 7 where we present an explicit order-preserving map $\mathcal{T}$ from $\mathcal{B}(n-2, d-1)$ to $\mathcal{S}_{1}(n, d)$ that should help to get a more concrete idea of the connections between higher Bruhat orders and higher Stasheff-Tamari orders. Furthermore, we relate some of the functorial constructions for higher Bruhat orders to similar constructions for higher Stasheff-Tamari orders.

In Section 6 we will recall the main definitions and results in the framework of higher Bruhat orders. Additionally, we answer a question posed by ZIEGLER [15] on the existence of an order-preserving embedding of $\mathcal{B}(n, k)$ into $\mathcal{B}(n+1, k+1)$ affirmatively.

The following three problems concerning the higher Stasheff-Tamari orders remain open:

- Is $\mathcal{S}_{1}(n, d)$ equal to $\mathcal{S}_{2}(n, d)$ ?

- Is $S_{1}(n, d)$ or $S_{2}(n, d)$ a lattice?

- Is $\mathcal{T}$ surjective; in particular is $\mathcal{T}$ the map suggested by KAPRANOV $\&$ VOEVODSKY?

Throughout this paper the following notation is used:

- For a set $L$ and " $<_{l}$ " a linear order on $L$, we denote by $L_{<_{l}}$ the set $L$ linearly ordered by " $<_{l}$."

- Numbers in brackets $\left(i_{1}, \ldots, i_{n}\right)$ denote the set $\left\{i_{1}, \ldots, i_{n}\right\}<$ which is linearly ordered by with $i_{\mathrm{v}}<i_{\mathrm{v}+1}$ for $v=1, \ldots, n-1$. 
- Let $L$ be a set. For a subset $S \subseteq L$ let $C S=\complement_{L} S$ be the complement $L \backslash S$ of $S$ in $L$.

- For a set $L$ and two sets $K$ and $K^{\prime}$ of subsets of $L$ such that $S \cap S^{\prime}=\varnothing$ for all $S \in K$ and $S^{\prime} \in K^{\prime}$ let $K * K^{\prime}:=\left\{S \cup S^{\prime}: S \in K, S^{\prime} \in K^{\prime}\right\}$ be the join of $K$ and $K^{\prime}$.

- For a set $K$ of subsets of $L$ and $S_{0} \in K$ the deletion of $S_{0}$ from $K$ is the set $K \backslash S_{0}:=\left\{S \in K: S \cap S_{0}=\varnothing\right\}$, and the contraction of $S_{0}$ in $K$ is the set $K / S_{0}:=\left\{S \backslash S_{0}: S \in K, S \supseteq S_{0}\right\}$.

- For integers $a<b$ the interval $[a, b]$ is the set $\{a, a+1, \ldots, b-1, b\}$ and $] a, b[$ is the set $\{a+1, \ldots, b-1\}$,

- $[n]$ denotes the interval $[1, n]$, and $] n[$ is the interval $] 1, n[$.

\section{A COMBINATORIAL FRAMEWORK FOR TRIANGULATIONS}

In this section we present a combinatorial concept of triangulations that is similar to that of DE LOERA [5]. Dealing with vertex labels when investigating triangulations is formally justified by the following considerations that are closely related to the theory of abstract simplicial complexes.

Definition 2.1. Let $\mathcal{L}$ be a finite set, the label set. A combinatorial $d$ simplex in $\mathcal{L}$ is a $(d+1)$-element-subset $S$ of $\mathcal{L}$. Its $(k+1)$-subsets are called $k$-faces of $S$, and its $d$-subsets facets of $S$.

If $\ell: \mathcal{L} \rightarrow \mathbb{R}^{N}$ is an injective function with $\ell(\mathcal{L})=: \mathcal{A}$, and $S \subset \mathcal{L}$ is a combinatorial $d$-simplex corresponding to affinely independent points then the convex hull $\sigma=\operatorname{conv} \ell(S)$ of $\ell(S)$ is the geometric $d$-simplex with vertex set vert $\sigma=\ell(S)$ and label set $\operatorname{lab}(\sigma)=S$ with respect to $\ell$, the labelling function.

A combinatorial simplicial complex in $\mathcal{L}$ is a set $K$ of combinatorial simplices in $\mathcal{L}$. Its $k$-simplices are the $k$-faces of its elements. (That is, we identify the usual abstract simplicial complexes with their set of inclusionmaximal faces.) A set $\Delta$ of geometric simplices $\sigma$ with the property that the set $\{\operatorname{lab}(\sigma): \sigma \in \Delta\}$ of label sets is a combinatorial simplicial complex, and that

$$
\operatorname{conv}(\operatorname{vert} \sigma \cap \operatorname{vert} \tau)=\sigma \cap \tau \quad \text { for all } \sigma, \tau \in \Delta,
$$

is a geometric simplicial complex.

A combinatorial simplicial complex $K^{\prime}$ is a combinatorial subcomplex of $K$ if all simplices of $K^{\prime}$ are faces of simplices in $K$. A geometric subcomplex is defined analogously.

For a combinatorial simplicial complex $K$ in $\mathcal{L}$ and a combinatorial simplex $S_{0}$ in $\mathcal{L}$ the combinatorial link of $S_{0}$ in $K$ is defined as

$$
\mathrm{lk}_{K}\left(S_{0}\right):=\left\{S \backslash S_{0}: S \in K, S_{0} \subseteq S\right\} ;
$$

the combinatorial star of $S_{0}$ in $K$ is defined by

$$
\mathrm{st}_{K}\left(S_{0}\right):=\left\{S \in K: S_{0} \subseteq S\right\},
$$


and the combinatorial antistar of $S_{0}$ in $K$ is the complex

$$
\operatorname{ast}_{K}\left(S_{0}\right):=\left\{S \in K: S \cap S_{0}=\varnothing\right\} .
$$

If $K$ is a combinatorial simplicial complex in $\mathcal{L}$ and $S_{0}$ is a combinatorial simplex in $\mathcal{L}^{\prime}$ where $\mathcal{L}$ and $\mathcal{L}^{\prime}$ are disjoint then the combinatorial join of $K$ and $S_{0}$ is the complex

$$
K * S_{0}:=\left\{S \cup S_{0}: S \in K\right\} .
$$

The convex hull conv $\mathcal{A}$ of $\mathcal{A}$ is a $d$-polytope if the affine hull of $\mathcal{A}$ is $\mathbb{R}^{d}$. For $\mathcal{A}^{\prime} \subset \mathcal{A}$ the polytope conv $\mathcal{A}^{\prime}$ is a facet of conv $\mathcal{A}$, if conv $\mathcal{A}^{\prime}$ is the $(d-1)$-dimensional intersection of $\mathcal{A}$ with a hyperplane $H$ such that one closed halfspace defined by $H$ contains conv $\mathcal{A}$. In this case the label set $\operatorname{lab}\left(\mathcal{A}^{\prime}\right)$ is a combinatorial facet of $\ell$. Note that the set of facets of a simplicial polytope (all facets are simplices) forms a simplicial complex.

If $Z=\left(Z^{+}, Z^{-}\right)$is a pair of disjoint inclusion minimal subsets $Z^{+}$and $Z^{-}$ of $\mathcal{L}$ with the property

$$
\operatorname{conv} \ell\left(Z^{+}\right) \cap \operatorname{conv} \ell\left(Z^{-}\right) \neq \varnothing
$$

then $Z$ is called a minimal combinatorial dependence in $\ell$, or - for short - a circuit of $\ell$. The set $\operatorname{supp}(Z)=Z^{+} \cup Z^{-}$is the support of $Z$.

The tripel $\mathcal{P}(\ell)=\left(\mathcal{L}, \mathcal{F}_{\ell}, Z_{\ell}\right)$, where $Z_{\ell}$ denotes the set of all circuits of $\ell$, and $\mathcal{F}_{\ell}$ is the set of all combinatorial facets of $\ell$, is the combinatorial polytope of $\ell$.

If $\Delta$ a geometric simplicial complex with vertices in $\mathcal{A}$ such that conv $\mathcal{A}=$ $\bigcup_{\sigma \in \Delta} \sigma$ then $\Delta$ is called a triangulation of $\mathcal{A}$. In this case the set $T$ of label sets of the simplices in $\Delta$ is a combinatorial triangulation of $\mathcal{P}(\ell)$.

We will sometimes call the geometric objects geometric interpretations of the corresponding combinatorial ones, which themselves are said to be combinatorial models for their geometric counterparts.

A combinatorial, label-based handling of triangulations is made possible by the following proposition. We present a complete elementary proof because this characterization is fundamental for this paper.

Proposition 2.2. Let $\mathcal{L}$ be a finite set and let $\ell: \mathcal{L} \rightarrow \mathbb{R}^{d}$ be injective with $\ell(\mathcal{L})=: \mathcal{A}$. Furthermore, let $\mathcal{P}(\ell)=\left(\mathcal{L}, \mathcal{F}_{\ell}, Z_{\ell}\right)$ be the combinatorial polytope of $\ell$. A non-empty subset $T$ of the $(d+1)$-subsets of $\mathcal{L}$ is a combinatorial triangulation of $\ell$ if and only if

(UP) for all $S \in T$ and all facets $F$ of $S$ either $F$ is contained in some $F^{\prime} \in \mathcal{F}_{\ell}$, or there is another simplex $S^{\prime} \in T$ such that $S^{\prime} \supset F$ (UnionProperty), and

(IP) there is no circuit $Z \in Z_{\ell}$ with $Z^{+} \subset S$ and $Z^{-} \subset S^{\prime}$ for combinatorial simplices $S, S^{\prime} \in T$ (Intersection-Property).

Proof. We first prove that (UP) and (IP) are necessary. Let $T$ be a combinatorial triangulation with respect to some geometric triangulation $\Delta$ of the point set $\mathcal{A}$ given by $\ell: \mathcal{L} \rightarrow \mathbb{R}^{d}$. Assume there is a combinatorial facet $F$ 
of some combinatorial $d$-simplex $S$ in $T$ that is not contained in $\mathcal{F}_{\ell}$, such that there is no other combinatorial $d$-simplex in $T$ containing $F$. Then the corresponding $(d-1)$-simplex $\tau:=\operatorname{conv} \ell(F)$ is contained in only one simplex $\sigma:=\operatorname{conv} \ell(S)$ of $\Delta$.

Let $H$ be a supporting hyperplane of $\tau$ such that its closed positive halfspace $H^{+}$contains $\sigma$. Let $q_{\tau}$ be the barycenter of $\tau$. Because $\tau$ is not a facet of $P=\operatorname{conv}(\mathcal{A})$ there is a point $x_{0}$ in $P$ lying in the open negative halfspace relint $\left(H^{-}\right)$. Connect $q_{\tau}$ and $x_{0}$ by a segment $I$. This segment is completely contained in $P$ since $P$ is convex.

$\Delta$ is a triangulation. Hence, there must be at least one $d$-simplex $\sigma_{x_{0}}$ that contains $x_{0}$. Either $\sigma_{x_{0}}$ contains $q_{\tau}$ or not. If it does then $\sigma_{x_{0}}$ must contain the complete $(d-1)$-simplex $\tau$ as a facet since $q_{\tau}$ lies in the relative interior of $\tau$ and the intersection of $\tau$ and $\sigma_{x_{0}}$ must be a face of both. But this is a contradiction.

If $\sigma_{x_{0}}$ does not contain $q_{\tau}$ then the segment $I$ intersects the boundary of $\sigma_{x_{0}}$ in a point $q_{x_{0}}$. Consider the mid-point $x_{1}$ of $q_{\tau}$ and $q_{x_{0}}$ on $I$. This point is neither contained in $\tau$ nor in $\sigma_{x_{0}}$. Since $I$ lies completely in $P$ there must be a new $d$-simplex $\sigma_{x_{1}}$ in $\Delta$ containing $x_{1}$. Because this procedure shows either a contradiction as above or an infinite sequence of $d$-simplices in $\Delta$, which is a contradiction, too. Hence, Property (UP) is necessary.

For the necessity of Property (IP) assume that there are combinatorial $d$ simplices $S$ and $S^{\prime}$ in $T$ and a circuit $Z=\left(Z^{+}, Z^{-}\right)$in $Z(n, d)$ such that $Z^{+}$is contained in $S$ and $Z^{-}$is contained in $S^{\prime}$. Then by the definition of circuits

$$
\operatorname{conv} \ell\left(Z^{+}\right) \cap \operatorname{conv} \ell\left(Z^{-}\right) \neq \varnothing,
$$

and their minimality implies that there are geometric simplices in $\Delta$, namely conv $\ell\left(Z^{+}\right)$and conv $\ell\left(Z^{-}\right)$the relative interiors of which intersect, a contradiction. Hence, Property (IP) is necessary as well.

Let $T$ be a collection of $(d+1)$-subsets of $\mathcal{L}$ (that is, $T \subseteq\left(\begin{array}{c}\mathcal{L} \\ d+1\end{array}\right)$ ) satisfying (UP) and (IP). Then $T$ gives rise to a set of geometric simplices $\Delta:=\{\operatorname{conv} \ell(S): S \in T\}$. We must show that every point in $P$ lies in at least one $d$-simplex $\sigma$ in $\Delta$ and that for every pair of simplices $\sigma$ and $\sigma^{\prime}$ we have $\operatorname{conv}\left(\right.$ vert $\sigma \cap$ vert $\left.\sigma^{\prime}\right)=\sigma \cap \sigma^{\prime}$.

Let $x$ be an arbitrary point in $P$. Since $T$ is non-empty we find a combinatorial $d$-simplex $S_{0}$ in $T$. Hence there is a simplex $\sigma_{0}:=\ell\left(S_{0}\right)$ in $\Delta$. Consider a segment $I$ from an inner point $x_{0}$ of $\sigma_{0}$ to $x$ that does not meet any $(d-2)$-simplex of $\Delta$. Such a line exists because of the concept of general position. This segment is completely contained in $P$ and meets exactly one facet $\tau$ of $\sigma_{0}$ unless $x \in \operatorname{relint}\left(\sigma_{0}\right)$. If this intersection point $q_{\tau}$ equals $x$ then we are done. Otherwise this facet is not a facet of $P$ because then $q_{\tau}$ is an interior point of $I$ and $I$ is contained in $P$. Hence the label set $F$ of $\tau$ is not in $\mathcal{F}_{\ell}$, and we find another combinatorial $d$-simplex $S_{1}$ in $T$ containing $F$ corresponding to a geometric $d$-simplex $\sigma_{1}$ containing $\tau$. The segment $I$ meets the interior of $\sigma_{1}$ because of the general position property of $I$. Choose an arbitrary point $x_{1}$ in $I \cap \operatorname{relint}\left(\sigma_{1}\right)$. Note that the distance between $x_{1}$ and $x$ is 
strictly smaller than the distance between $x_{0}$ and $x$. Therefore, by repeating this procedure we will reach a $d$-simplex $\sigma_{r}$ lying in $\Delta$ and containing $x$.

Now assume that there are geometric $d$-simplices $\sigma$ and $\sigma^{\prime}$ in $\Delta$ with label sets $S$ respectively $S^{\prime}$ in $T$ and $\operatorname{conv}\left(\right.$ vert $\sigma \cap$ vert $\left.\sigma^{\prime}\right) \subset \sigma \cap \sigma^{\prime}$. Since $\sigma \supseteq \sigma \cap \sigma^{\prime}$ and $\sigma^{\prime} \supseteq \sigma \cap \sigma^{\prime}$ there are inclusion-minimal faces $\tau$ of $\sigma$ and $\tau^{\prime}$ of $\sigma^{\prime}$ with $\operatorname{conv}\left(\operatorname{vert} \tau \cap \operatorname{vert} \tau^{\prime}\right) \supseteq \sigma \cap \sigma^{\prime}$. From the minimality assumption we get relint $(\tau) \cap \operatorname{relint}\left(\tau^{\prime}\right) \neq \varnothing$, hence by Radon's Theorem there are minimal, vertex-disjoint faces $\rho$ of $\tau$ and $\rho^{\prime}$ of $\tau^{\prime}$ with relint $(\rho) \cap \operatorname{relint}\left(\rho^{\prime}\right) \neq \varnothing$. Set $Z^{+}:=\operatorname{lab}(\rho)$ and $Z^{-}:=\operatorname{lab}\left(\rho^{\prime}\right)$. Then $Z^{+}$and $Z^{-}$are disjoint and $\operatorname{conv}\left(\ell\left(Z^{+}\right)\right) \cap \operatorname{conv}\left(\ell\left(Z^{-}\right)\right) \neq \varnothing$. Hence $\left(Z^{+}, Z^{-}\right)$lies in $Z_{\ell}$, and $Z^{+}$is contained in $S$ and $Z^{-}$is contained in $S^{\prime}$, but this contradicts the assumption that $T$ has Property (IP).

Pairs of simplices with property (IP) are called admissible.

\section{CYCLIC POLYTOPES}

In this section we recall the basic definitions and theorems related to cyclic polytopes in a combinatorial language.

Definition 3.1. Let $\mathcal{L}$ be a linearly ordered set, and let $t: \mathcal{L} \rightarrow \mathbb{R}, i \mapsto t_{i}$ be a strictly monotone function.

The $d$-dimensional cyclic polytope $C(\mathcal{L}, d, t)$, labelled by $\mathcal{L}$, parametrized by $t$ is the convex hull of the points $v_{d}\left(t_{1}\right), \ldots, v_{d}\left(t_{n}\right)$ with

$$
\mathrm{v}_{d}(x):=\left(x, x^{2}, \ldots, x^{d}\right) \in \mathbb{R}^{d} .
$$

For simplicity we set $C(n, d, t):=C([n], d, t)$.

The main reason for the fact that triangulations of cyclic polytopes can be treated effectively in a purely combinatorial way are the following wellknown properties that follow from the special structure of Vandermondedeterminants.

The first one - Gale's famous Evenness Criterion - characterizes the set $\mathcal{F}_{v_{d} \text { ot }}$ of all combinatorial facets of $C(\mathcal{L}, d, t)$. The following notion allows us to state that criterion in a compact way.

Definition 3.2. Let $L$ be a linearly ordered set and $S$ a subset of $L$. An element $s_{0} \in \complement S$ is an even gap in $S$ if \# $\left\{s \in S: s>s_{0}\right\}$ is even, otherwise it is an odd gap.

Theorem 3.3 (Gale's Evenness Criterion [9]). An ordered subset $F$ of the vertex set of the cyclic polytope $C(\mathcal{L}, d, t)$ is a facet if and only if between any two vertices not in $F$ there is an even number of vertices in $F$. Equivalently, $F$ is a facet of $C(\mathcal{L}, d, t)$ if and only if either all gaps in $F$ are even or all gaps in $F$ are odd.

The second one describes the form of those sets of vertices of $C(\mathcal{L}, d, t)$ the convex hulls of which intersect in the relative interior of both. Hence this determines $C_{\mathrm{v}_{d} \mathrm{t}}$. 
Theorem 3.4. [4] The circuits of $C(\mathcal{L}, d, t)$ are the alternating $(d+2)$-subsets of $\mathcal{L}, i$. e., the pairs $\left(Z^{o}, Z^{e}\right)$ and $\left(Z^{e}, Z^{o}\right)$, where $Z^{o}$ is the set of odd elements $\left(z_{1}, z_{3}, z_{5}, \ldots\right)$, and $Z^{e}$ is the set of even elements $\left(z_{2}, z_{4}, z_{6}, \ldots\right)$ of $Z=\left(z_{1}, \ldots, z_{d+2}\right)$.

The combinatorial polytopes $P\left(v_{d} \circ t\right)$ are identical for all $t$ because the strictly monotone function $t$ does not affect the assertions of these criteria. This means that the combinatorial study of triangulations of cyclic polytopes with any parametrization is equivalent to the investigation of combinatorial triangulations of the combinatorial polytopes $\mathcal{P}\left(\mathrm{v}_{d} \circ t\right)$.

Definition 3.5. The combinatorial polytope $C(\mathcal{L}, d):=\mathcal{P}\left(\mathrm{v}_{d} \circ t\right)$ of $\mathrm{v}_{d} \circ t$ : $\mathcal{L} \rightarrow \mathbb{R}^{d}$ is called the cyclic d-polytope with vertices labelled by $\mathcal{L}$. The set of its combinatorial facets is denoted by $\mathcal{F}(\mathcal{L}, d)$, the set of its circuits is written as $Z(\mathcal{L}, d)$. Those combinatorial facets with only odd gaps are the upper facets the set of which is denoted by $\mathcal{F}^{u}(\mathcal{L}, d)$, those with only even gaps are the lower facets of $C(\mathcal{L}, d)$, denoted by $\mathcal{F}^{l}(\mathcal{L}, d)$.

The set of circuits $Z$ with maximal element $z_{d+2}$ in $Z^{+}$is denoted by $Z^{+}(n, d)$, the set of circuits having their maximal element in $Z^{-}$is written as $Z^{-}(n, d)$. The cyclic polytope labelled by $[n]$ is denoted by $C(n, d)$.

Note that in odd dimensions there are polytopes that have the same face lattice as $C(n, d, t)$ but a different circuit structure (see [4]); this leads to completely different triangulations.

Remark 3.6 (Geometric Meaning, see Figure 1). Consider for some strictly monotone $t:[n] \rightarrow \mathbb{R}$ the projection

$$
p=p(n, d):\left\{\begin{aligned}
C(n, d+1, t) & \rightarrow C(n, d, t), \\
\left(x_{1}, \ldots, x_{d}, x_{d+1}\right) & \mapsto\left(x_{1}, \ldots, x_{d}\right) .
\end{aligned}\right.
$$

Moreover, consider for some geometric triangulation $\Delta$ of $C(n, d, t)$ the unique piecewise linear section (linear on each simplex $\sigma \in \Delta$ )

$$
s_{\Delta}:\left\{\begin{aligned}
& C(n, d, t) \rightarrow C(n, d+1, t), \\
& \sigma \stackrel{\text { linear }}{\mapsto} \operatorname{conv}\left(v_{d+1} \circ t(\operatorname{lab}(\sigma))\right), \quad \forall \sigma \in \Delta .
\end{aligned}\right.
$$

Then any triangulation $\Delta$ of $C(n, d, t)$ can be recovered from its characteristic section $s_{\Delta}$.

The upper facets $\mathcal{F}^{u}(n, d+1)$ of $C(n, d+1)$ are the sets of those facets of $C(n, d+1, t)$ that can be seen from a point in $\mathbb{R}^{d+1}$ with very large positive $(d+1)$-st coordinate (geometric upper facets of $C(n, d+1, t))$, the lower facets $\mathcal{F}^{u}(n, d+1)$ label the sets of those facets of $C(n, d+1, t)$ that can be seen from a point in $\mathbb{R}^{d+1}$ with very large negative $(d+1)$-st coordinate (geometric lower facets of $C(n, d+1, t)$ ). The geometric upper (respectively lower) facets project down to $C(n, d, t)$ without overlapping. Therefore their projections define geometric triangulations of $C(n, d, t)$.

The support $\operatorname{supp}(Z)$ of any circuit $Z=\left(Z^{+}, Z^{-}\right)$in $C(n, d)$ corresponds to the label set of a unique $(d+1)$-simplex in $C(n, d+1, t)$ where its set 
10

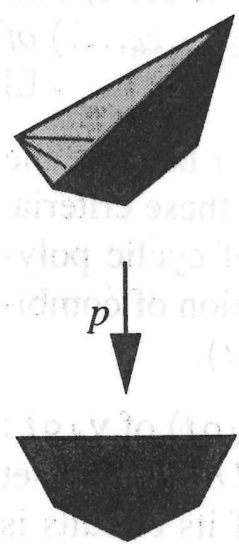

JÖRG RAMBAU
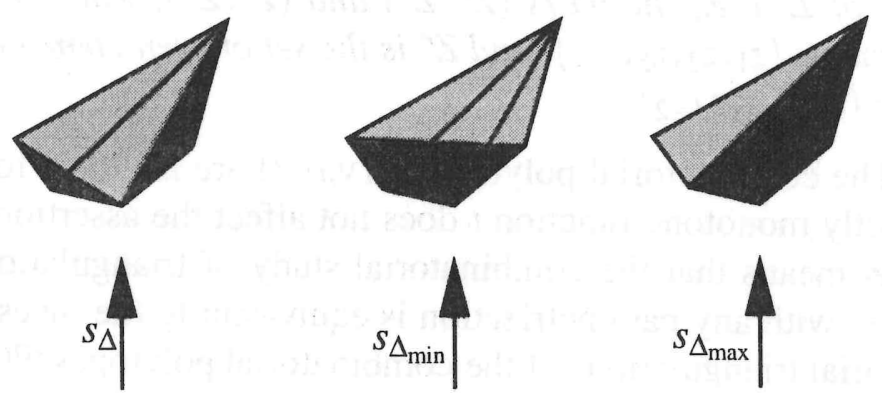

$s_{\Delta_{\max }} \uparrow$
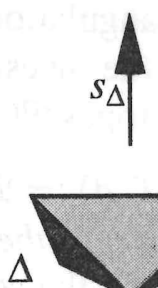

$\Delta$
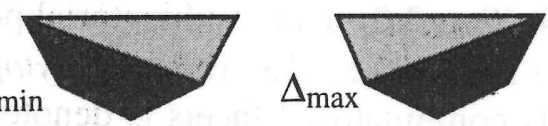

FIGURE 1. The canonical projection $p: C(5,3) \rightarrow C(5,2)$ and sections corresponding to triangulations of $C(5,2)$.

of geometric upper facets belongs to the elements of the star of the positive part $Z^{+}$in $\operatorname{supp}(Z)$, and its set of geometric lower facets corresponds to the elements of the star of the negative $\operatorname{part} Z^{-}$in $\operatorname{supp}(Z)$.

Lemma 3.7. (Elementary Facts)

(i) $\mathcal{F}^{l}(n, d+1)$ and $\mathcal{F}^{u}(n, d+1)$ are combinatorial triangulations of the cyclic polytope $C(n, d)$.

(ii) Every facet in $\mathcal{F}^{u}(n, d)$ contains $n$.

(iii) If a pair of simplices $S_{1}$ and $S_{2}$ is not admissible then there exists a circuit in $Z(n, d)$ with maximal element $z_{d+2}=\max \left(S_{1} \cup S_{2}\right)$.

(iv) If $a(d-1)$-simplex $F$ is the common facet of the admissible pair $\left(S_{1}, S_{2}\right)$ then $S_{1} \backslash F$ lies in an odd gap of $F$ and $S_{1} \backslash F$ lies in an even gap of $F$, or vice versa.

Remark 3.8. The circuits of $C(n, d)$ can be visualized in a table that consists of columns numbered from 1 to $n$ and rows corresponding to $Z^{+}$and $Z^{-}$, where a star "*" in column $i$ and row $Z^{\varepsilon}$ means that $i \in Z^{\varepsilon}, \varepsilon \in\{+,-\}$. The stars can then be connected by a zig-zag-path with $(d+2)$ nodes. For example, if $n=6, d=3$, and $Z=((1,3,5),(2,4))$ we get the table

\begin{tabular}{|l||c|c|c|c|c|c|}
\hline & 1 & 2 & 3 & 4 & 5 & 6 \\
\hline \hline$Z^{+}$ & $*$ & & $*$ & & $*$ & \\
\hline$Z^{-}$ & & $*$ & & $*$ & & \\
\hline
\end{tabular}

If the rows are filled with stars corresponding to two simplices then these two simplices are admissible if and only if each zig-zag-path connects at most $(d+1)$ stars. For instance if $n=6, d=3, S=(1,3,4,5)$, and $S^{\prime}=$ $(2,3,4,6)$ the table looks as follows:

\begin{tabular}{|c||c|c|c|c|c|c|}
\hline & 1 & 2 & 3 & 4 & 5 & 6 \\
\hline \hline$S$ & $*$ & & $*$ & $*$ & $*$ & \\
\hline$S^{\prime}$ & & $*$ & $*$ & $*$ & & $*$ \\
\hline
\end{tabular}


The reader will easily find a zig-zag-path connecting even $6>d+2$ stars, showing that $S, S^{\prime}$ is not an admissible pair.

Obviously all $C(\mathcal{L}, d)$ with $\# \mathcal{L}=n$ are isomorphic to $C(n, d)$. From now on we are exclusively dealing with combinatorial triangulations of $C(n, d)$, and we will leave out the "combinatorial" attribute whenever this is not confusing.

The following Propositions - consequences of Theorems 3.3 and 3.4 - relate cyclic polytopes with different parameters. We use the notation $F=\left(f_{1}, \ldots, f_{d}\right)$ for $F \in \mathcal{F}(n, d)$ and $Z=\left(z_{1}, \ldots, z_{d+2}\right)$ for $Z \in Z(n, d)$.

Proposition 3.9. (Functorial Facet Properties)

$$
\begin{aligned}
\mathcal{F}^{u}(n+1, d+1)= & \mathcal{F}^{l}(n, d) *\{n+1\}, \\
\mathcal{F}^{l}(n+1, d+1)= & \mathcal{F}^{u}(n, d) *\{n+1\} \\
& \cup\left\{F \backslash n \cup\{j, j+1\}: F \in \mathcal{F}^{u}(n, d), j \in\right] f_{d-1}, n[\}, \\
\mathcal{F}^{u}(n-1, d-1)= & \operatorname{lk}_{\mathcal{F}^{l}(n, d)}(n), \\
\mathcal{F}^{l}(n-1, d-1)= & \operatorname{lk}_{\mathcal{F}^{u}(n, d)}(n), \\
\mathcal{F}^{u}(n-1, d)= & \operatorname{lk}_{\mathcal{F}^{u}(n, d)}(n) *\{n-1\}, \\
\mathcal{F}^{l}(n-1, d) & =\operatorname{ast}_{\mathcal{F}^{l}(n, d)}(n) .
\end{aligned}
$$

Proposition 3.10. (Functorial Circuit Properties)

$$
\begin{aligned}
Z^{+}(n+1, d+1) & =\left\{\left(Z^{+} \cup\{j\}, Z^{-}\right):\left(Z^{+}, Z^{-}\right) \in Z^{-}(n, d), j>z_{d+2}\right\}, \\
Z^{-}(n+1, d+1) & =\left\{\left(Z^{+}, Z^{-} \cup\{j\}\right):\left(Z^{+}, Z^{-}\right) \in Z^{+}(n, d), j>z_{d+2}\right\}, \\
Z^{+}(n-1, d-1) & =\left\{\left(Z^{+}, Z^{-} \backslash z_{d+2}\right):\left(Z^{+}, Z^{-}\right) \in Z^{-}(n, d)\right\}, \\
Z^{-}(n-1, d-1) & =\left\{\left(Z^{+} \backslash z_{d+2}, Z^{-}\right):\left(Z^{+}, Z^{-}\right) \in Z^{+}(n, d)\right\}, \\
Z^{+}(n-1, d) & =\left\{\left(Z^{+}, Z^{-}\right) \in Z^{+}(n, d): n \notin \operatorname{supp}(Z)\right\}, \\
Z^{-}(n-1, d) & =\left\{\left(Z^{+}, Z^{-}\right) \in Z^{-}(n, d): n \notin \operatorname{supp}(Z)\right\} .
\end{aligned}
$$

The following proposition is the combinatorial description for the geometric connection provided by the projection $p(n, d)$ between $(d+1)$-simplices in $C(n, d, t)$ and the minimal affine dependencies in $C(n, d, t)$

Proposition 3.11. (Functorial Circuit-Facet-Relations)

For $Z \in Z(n, d)$ and $\operatorname{supp}(Z)$ considered as a simplicial complex we have

$$
\begin{aligned}
& \mathrm{st}_{\operatorname{supp}(Z)}\left(Z^{+}\right)=\mathcal{F}^{u}(\operatorname{supp}(Z), d+1), \\
& \mathrm{st}_{\operatorname{supp}(Z)}\left(Z^{-}\right)=\mathcal{F}^{l}(\operatorname{supp}(Z), d+1) .
\end{aligned}
$$

\section{SPECIAL TRIANGULATIONS OF CYCLIC POLYTOPES}

In this section we show nice functorial constructions of triangulations of cyclic polytopes. 
Definition 4.1. For a set $T$ of $(d+1)$-subsets of $[n]$ define

$$
\begin{aligned}
& \hat{T}:=T *\{n+1\} \\
& \cup\left\{S \backslash s_{d+1} \cup\{j, j+1\}: S=\left(s_{1}, \ldots, s_{d+1}\right) \in T, j \in\right] s_{d}, s_{d+1}[\}, \\
& \text { (extension) } \\
& T / n:=\operatorname{lk}_{T}(n), \\
& T \backslash n:=\operatorname{ast}_{T}(n) \cup \operatorname{ast}_{\mathbf{l k}_{T}(n)}(n-1) *\{n-1\} .
\end{aligned}
$$

Theorem 4.2. Let $T \in S(n, d)$. Then the following hold:

(i) $\hat{T}$ is a triangulation of $C(n+1, d+1)$,

(ii) $T / n$ is a triangulation of $C(n-1, d-1)$,

(iii) $T \backslash n$ is a triangulation of $C(n-1, d)$.

Proof. For each assertion we verify the Union-Property (UP) and the Intersection-Property (IP) of Proposition 2.2. Recall that we have to show roughly speaking - that

- all simplices are pairwise admissible, and that

- each facet of a simplex is either a facet of the cyclic polytope or appears in at least one other simplex.

The reader may get a picture from the proof by inspecting the tables suggested in Remark 3.8, using that circuits correspond to zig-zag-paths and facets to sets with only even or only odd gaps.

Part (ii) is true because the link of a triangulation of any polytope at some vertex triangulates the corresponding vertex figure, and for cyclic polytopes this vertex figure is cyclic with the correct parameters. This follows from Propositions 3.9 and 3.10 and well-known properties of vertex figures (see, e. g., GRÜNBAUM [9]).

The proof of (UP) (i). The following abbreviations are used:

$$
\begin{aligned}
& A:=T *\{n+1\}, \\
& B:=\left\{S \backslash s_{d+1} \cup\{j, j+1\}: S \in T, j \in\right] s_{d}, s_{d+1}[\} .
\end{aligned}
$$

Let $F=\left(f_{1}, \ldots, f_{d+1}\right)$ be a facet of a simplex $S$ in $A \backslash \mathcal{F}(n+1, d+1)$.

The case $f_{d+1}=n+1$. By Proposition 3.9, $F \backslash n+1 \notin \mathcal{F}(n, d)$ because otherwise $(F \backslash n+1) \cup\{n+1\}$ is a facet of $C(n+1, d+1)$. Since $T$ has the Union-Property there must be a simplex $F^{\prime} \in T$ with $F \backslash n+1 \subset F^{\prime}$ and $F^{\prime} \neq F$. Hence

$$
F \subset \underbrace{F^{\prime} \cup n+1}_{\begin{array}{c}
\neq S \\
\text { since } F^{\prime} \neq F
\end{array}} \in \hat{T} .
$$

- The case $F \in T, f_{d+1}-f_{d}>1$. Then

$$
F \subset \underbrace{F \backslash f_{d+1} \cup\left\{f_{d+1}-1, f_{d+1}\right\}}_{\begin{array}{c}
\neq S \\
\text { since } n+1 \in S
\end{array}} \in \hat{T} .
$$




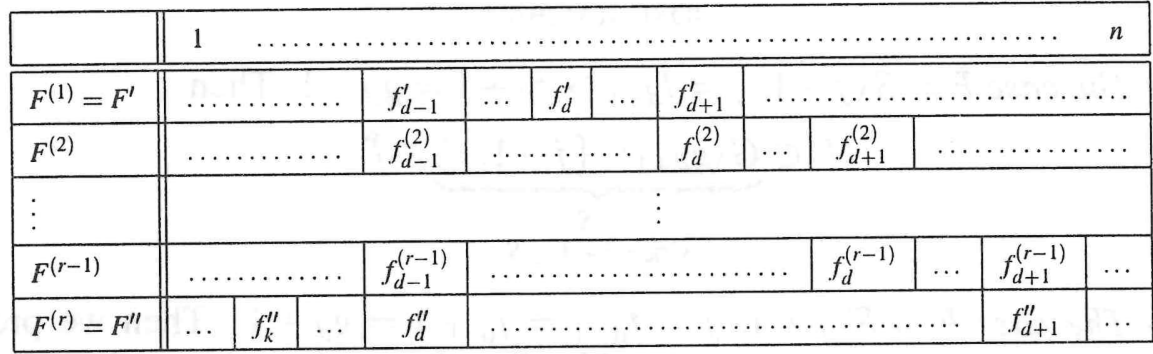

TABLE 1. The expansion of $F^{\prime}$ in $T$.

The case $F \in T, f_{d+1}-f_{d}=1$. By Proposition 3.9, $F \backslash f_{d} \notin \mathcal{F}(n, d)$ because either $f_{d+1}$ is an inner singleton in $F \backslash f_{d}$ or $f_{d+1}=n$ with the consequence that $\left(F \backslash f_{d}\right) \backslash n \cup\{n-1, n\}=F$ is a facet of $C(n+1, d+1)$. The Union-Property in $T$ leads to the existence of a simplex $F^{\prime}=\left(f_{1}^{\prime}, \ldots, f_{d+1}^{\prime}\right)$ in $T$ with $F \backslash f_{d} \subset F^{\prime}$ and $F^{\prime} \neq F$. The Intersection-Property in $T$ implies either

$$
f_{d+1}^{\prime}=f_{d+1}, \quad f_{d-1}^{\prime}=f_{d}
$$

or that

$$
f_{d+1}^{\prime}>f_{d+1}, \quad \quad f_{d-1}^{\prime}=f_{d-1} .
$$

(Compare Lemma 3.7(iv).)

In the first case $(*)$ we get

$$
F \subset \underbrace{F^{\prime} \backslash f_{d+1}^{\prime} \cup\left\{f_{d+1}-1, f_{d+1}\right\}}_{\begin{array}{c}
\neq S \\
\text { since } n+1 \in S
\end{array}} \in \hat{T} .
$$

In the second case (**) we know that $F^{\prime} \backslash f_{d}^{\prime} \notin \mathcal{F}(n, d)$. Performing the same steps for $F^{\prime} \backslash f_{d}^{\prime}$ yields a finite sequence $F^{\prime}=F^{(1)}, F^{(2)}, \ldots, F^{(r)}=F^{\prime \prime}$ (where $F^{(\mu)}=\left(f_{1}^{(\mu)}, \ldots, f_{d+1}^{(\mu)}\right)$ for $\mu \in\{1, \ldots, r\}$ ) of simplices in $T$ with

$$
\begin{gathered}
f_{d+1}^{\prime \prime}=f_{d+1}^{(r-1)}>f_{d+1}^{(r-2)}>\cdots>f_{d+1}, \\
f_{d}^{\prime \prime}=f_{d-1}^{(r-1)}=f_{d-1}^{(r-2)}=\cdots=f_{d-1}<f_{d}=f_{d+1}-1,
\end{gathered}
$$

where at step $(r)$ we end up in case $(*)$ because case $(* *)$ can occur at most $n-f_{d+1}$ times. This leads to

$$
F \subset \underbrace{F^{\prime \prime} \backslash f_{d+1}^{\prime \prime} \cup\left\{f_{d+1}-1, f_{d+1}\right\}}_{\begin{array}{c}
\neq S \\
\text { since } n+1 \in S
\end{array}} \in \hat{T} .
$$

For further use we refer to this sequence as the expansion of $F^{\prime}$.

Now let $F=\left(f_{1}, \ldots, f_{d+1}\right)$ be a facet of the simplex $S=G \backslash g_{d+1} \cup\{j, j+$ $1\}$ in $B$, such that $F$ is not a facet of $C(n+1, d+1)$, with $G=\left(g_{1}, \ldots, g_{d+1}\right)$ in $T$. 
The case $F=S \backslash j+1, j=f_{d+1}>f_{d}+1=g_{d}+1$. Then

$$
F \subset \underbrace{G \backslash g_{d+1} \cup\{j-1, j\}}_{\begin{array}{c}
\neq S \\
\text { since } j+1 \in S
\end{array}} \in \hat{T} .
$$

The case $F=S \backslash j+1, j=f_{d+1}=f_{d}+1=g_{d}+1$. Then we proceed as follows. $G \backslash g_{d}$ is not in $\mathcal{F}(n, d)$. Hence there is another simplex $G^{\prime}=\left(g_{1}^{\prime}, \ldots, g_{d+1}^{\prime}\right)$ in $T$ with $G \backslash g_{d} \subset G^{\prime}$. Consider the expansion $G^{\prime}=G^{(1)}, G^{(2)}, \ldots, G^{(r)}=G^{\prime \prime}$ of $G^{\prime}$. We have

$$
g_{d+1}^{\prime \prime} \geq f_{d+1}, \quad g_{d}^{\prime \prime}=f_{d-1}<f_{d}=f_{d+1}-1,
$$

and therefore

$$
F \subset \underbrace{G^{\prime \prime} \backslash g_{d+1}^{\prime \prime} \cup\left\{f_{d+1}-1, f_{d+1}\right\}}_{\text {since } j+1 \in S, j+1>f_{d+1}} \in \hat{T} .
$$

The case $F=S \backslash j, j+1<g_{d+1}$. Then

$$
F \subset \underbrace{G \backslash g_{d+1} \cup\{j+1, j+2\}}_{\text {since } j \in S, j>g_{d}} \in \hat{T} .
$$

The case $F=S \backslash j, j+1=g_{d+1}$. Then

$$
F=G \subset \underbrace{G \cup\{n+1\}}_{\begin{array}{c}
\neq S \\
\text { since } n+1 \notin S
\end{array}} \in \hat{T} .
$$

- The case $F=S \backslash g_{i}, 1 \leq i \leq d$. In this case $G \backslash g_{i}$ is not in $\mathcal{F}(n, d)$ because otherwise $\left(G \backslash g_{i}\right) \backslash g_{d+1} \cup\{j, j+1\}=F$ is a facet of $C(n+1, d+1)$ by Proposition 3.9. Hence we find a simplex $H=\left(h_{1}, \ldots, h_{d+1}\right)$ in $T$ with $G \backslash g_{i} \in H$ and $H \neq G$.

(*) If $h_{d+1}=g_{d+1}$ and $h_{d}<j$ then

$$
F \subset \underbrace{H \backslash h_{d+1} \cup\{j, j+1\}}_{\text {since } H \neq G, h_{d+1}=g_{d+1}} \in \hat{T} .
$$

(**) If $h_{d+1}=g_{d+1}$ and $h_{d}=j$ then either $h_{d+1}=j+1$ and thus $F=H$, or $h_{d+1}>j+1$, whence

$$
\begin{aligned}
& F=H \subset \underbrace{H \cup\{n+1\}}_{\neq S} \in \hat{T} \quad \text { in the first case, } \\
& \text { since } n+1 \notin S \\
& F \subset \underbrace{H \backslash h_{d+1} \cup\{j+1, j+2\}}_{\begin{array}{c}
\neq S \\
\text { since } j+2 \notin S
\end{array}} \in \hat{T} \quad \text { in the second case. }
\end{aligned}
$$

(***) If $h_{d+1}=g_{d+1}$ and $h_{d}>j$ then $h_{d}>g_{d}+1$ and hence $h_{d}-h_{d-1}>$ $g_{d}+1-g_{d}=1$. Therefore $H \backslash h_{d+1}$ is not in $\mathcal{F}(n, d)$ because $h_{d}$ is an inner 


\begin{tabular}{|c|c|c|c|c|c|c|c|c|}
\hline & 1 & & $\cdots$ & $j$ & $\ldots \ldots$ & $\ldots \ldots \ldots \ldots$ & $\cdots$ & $\ldots \ldots$ \\
\hline$H^{(1)}=H^{\prime}$ & $\ldots$ & $h_{d-1}^{\prime}$ & \multicolumn{4}{|c|}{ (. } & $h_{d}^{\prime}$ & $h_{d+1}^{\prime}$ \\
\hline$H^{(2)}$ & $\ldots$ & $h_{d-1}^{(2)}$ & \multicolumn{3}{|c|}{ 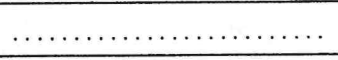 } & \begin{tabular}{l|l}
$\ldots$ & $h_{d}^{(2)}$ \\
\end{tabular} & $h_{d+1}^{(2)}$ & 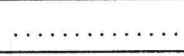 \\
\hline$\vdots$ & & & & & & \\
\hline$H^{(r-1)}$ & $\ldots$ & $h_{d-1}^{(r-1)}$ & \multicolumn{2}{|l|}{$\ldots$} & $h_{d}^{(r-1)}$ & $h_{d+1}^{(r-1)}$ & \multicolumn{2}{|c|}{ (n. } \\
\hline$H^{(r)}=H^{\prime \prime}$ & $\ldots$ & $h_{d-1}^{\prime \prime}$ & $h_{d}^{\prime \prime}$ & $\ldots$ & $h_{d+1}^{\prime \prime}$ & & & \\
\hline
\end{tabular}

TABLE 2. The compression of $H^{\prime}$ in $T$.

singleton. This implies that there is a simplex $H^{\prime}=\left(h_{1}^{\prime}, \ldots, h_{d+1}^{\prime}\right)$ in $T$ with $H \backslash h_{d+1} \subset H^{\prime}$. The Intersection-Property in $T$ leads to

$$
h_{d+1}^{\prime}=h_{d}>j, \quad h_{d}^{\prime}<h_{d} .
$$

Performing the above step with $H^{\prime}$ instead of $H$ induces a finite sequence (the compression of $H^{\prime}$ ) $H^{\prime}=H^{(1)}, H^{(2)}, \ldots, H^{(r)}=H^{\prime \prime}$ where for $H^{\prime \prime}$ case $(*)$ or case $(* *)$ must occur because the $d$-th element decreases monotonely. Then

$$
h_{d}^{\prime \prime} \leq j<h_{d}^{(r-1)}=h_{d+1}^{\prime \prime},
$$

and the constructions in (*) and (**) work with $H^{\prime \prime}$ instead of $H$ as well.

$(* * * *)$ If $h_{d+1}>g_{d+1}$ then $H \backslash h_{d}$ is not a facet of $C(n, d)$, i. e., we find a simplex $H^{\prime}=\left(h_{1}^{\prime}, \ldots, h_{d+1}^{\prime}\right)$ in $T$ with $H \backslash h_{d} \subset H^{\prime}$ and $H^{\prime} \neq H$, and we can finish the proof by using the expansion of $H^{\prime}$.

The proof of (IP) (i). We must show that any pair of simplices $(R, S)$ with $R=\left(r_{1}, \ldots, r_{d+2}\right)$ and $S=\left(s_{1}, \ldots, s_{d+2}\right)$ in $\hat{T}$ is admissible. Without loss of generality $\max (R \cup S) \in R$. There are three different cases:

- The case $R \in A, S \in A$. It is well-known that a pyramid over a simplicial complex is again a simplicial complex, i. e., it has the Intersection-Property.

The case $R \in B, S \in B$. There exist $R^{\prime}=\left(r_{1}^{\prime}, \ldots, r_{d+1}^{\prime}\right)$ and $S^{\prime}=$ $\left(s_{1}^{\prime}, \ldots, s_{d+2}^{\prime}\right)$ in $T$ such that

$$
\begin{array}{rlrl}
R & =: R^{\prime} \backslash r_{d+1}^{\prime} \cup\{j, j+1\}, & & r_{d}^{\prime}<j<r_{d+1}^{\prime}, \\
S=: S^{\prime} \backslash s_{d+1}^{\prime} \cup\{k, k+1\}, & & s_{d}^{\prime}<k<s_{d+1}^{\prime} .
\end{array}
$$

Without loss of generality, $j \geq k$. Assume $(R, S)$ is not admissible. Then, by Lemma 3.7, there exists a circuit $Z \in Z^{+}(n+1, d+1)$ with $\operatorname{supp}(Z)=$ $\left(z_{1}, \ldots, z_{d+3}\right)$ and

$$
Z^{+} \subset R, \quad Z^{-} \subset S, \quad z_{d+3}=r_{d+2}=j+1 .
$$

From Proposition 3.10 it follows that $Z^{\prime}:=\left(Z^{+} \backslash z_{d+3}, Z^{-}\right)$is a circuit in $Z^{-}(n, d)$ with

$$
\left(Z^{\prime}\right)^{+} \subset R \backslash\{j+1\}, \quad\left(Z^{\prime}\right)^{-} \subset S, \quad z_{d+2}^{\prime} \leq k+1 \leq s_{d+1}^{\prime} .
$$


Hence $z_{d+1}^{\prime}<j$ and $z_{d}^{\prime}<k$. Therefore

$$
\left(Z^{\prime}\right)^{+} \subset R^{\prime}, \quad\left(Z^{\prime}\right)^{-} \backslash z_{d+2}^{\prime} \cup s_{d+1}^{\prime} \subset S^{\prime} .
$$

But then

$$
Z^{\prime \prime}:=(\underbrace{\left(Z^{\prime}\right)^{+}}_{\subset R^{\prime}}, \underbrace{\left(Z^{\prime}\right)^{-} \backslash z_{d+2}^{\prime} \cup s_{d+1}^{\prime}}_{\subset S^{\prime}})
$$

is a circuit in $Z^{-}(n, d)$ showing that $\left(R^{\prime}, S^{\prime}\right)$ is not admissible, contradiction.

The case $R \in A, S \in B$. There exist $R^{\prime}=\left(r_{1}^{\prime}, \ldots, r_{d+2}^{\prime}\right)$ and $S^{\prime}=$ $\left(s_{1}^{\prime}, \ldots, s_{d+2}^{\prime}\right)$ in $T$ with

$$
\begin{aligned}
& R=: R^{\prime} \cup\{n+1\}, \\
& S=: S^{\prime} \backslash s_{d+1}^{\prime} \cup\{k, k+1\}, \quad s_{d}^{\prime}<k<s_{d+1}^{\prime} .
\end{aligned}
$$

Assume again that $(R, S)$ is not admissible. Let $Z \in Z^{+}(n+1, d+1)$ be a circuit with $\operatorname{supp}(Z)=\left(z_{1}, \ldots, z_{d+3}\right)$ such that

$$
Z^{+} \subset R, \quad Z^{-} \subset S, \quad z_{d+3}=r_{d+2}=n+1 .
$$

Then

$$
Z^{\prime}:=(\underbrace{Z^{+} \backslash n+1}_{\subset R^{\prime}}, \underbrace{Z^{-} \backslash z_{d+2} \cup s_{d+1}^{\prime}}_{\subset S^{\prime}})
$$

is a circuit in $Z^{-}(n, d)$ showing that $\left(R^{\prime}, S^{\prime}\right)$ is not admissible, contradiction. The proof of (UP) (iii). In order to simplify notation we set

$$
\begin{aligned}
& A:=\operatorname{ast}_{T}(n), \\
& B:=\operatorname{ast}_{\mathbf{l}_{T}(n)}(n-1) *\{n-1\} .
\end{aligned}
$$

We bring some known facts into a useful form:

(a) Let $F$ be a facet of $C(n-1, d-1)$ that does not contain $n-1$. Then $(F, n-1)$ is a facet of $C(n-1, d)$.

(b) Let $F$ be a facet of $C(n, d)$ that does not contain $n$ then $F$ is a facet of $C(n-1, d)$.

(c) $\operatorname{st}_{T}(n) \cup \operatorname{ast}_{T}(n)=T, \mathrm{st}_{T}(n) \cap \operatorname{ast}_{T}(n)=\mathrm{lk}_{T}(n)$.

Because of (c) all boundary facets of $A$ are contained in $\mathrm{lk}_{T}(n)$ or are facets of $C(n, d)$ that do not contain $n$. Then by (b) all boundary facets of $A$ that are not facets of $C(n-1, d)$ are contained in $\mathrm{lk}_{T}(n)$. Now let $F$ be an element of $1 \mathrm{k}_{T}(n)$ but not a facet of $C(n-1, d)$. If $n-1 \notin F$ then $(F, n-1) \in T \backslash n$. If $n-1 \in F$ then by (a) we know that $F \backslash(n-1)$ is not a facet of $C(n, d) / n$. Hence there is a simplex $S$ in $\operatorname{ast}_{l_{T}(n)}(n-1)$ that containes $F \backslash(n-1)$ and therefore $F \subset(S, n-1) \in T \backslash n$.

Now let $F$ be a facet in $B$ that is not in $\mathcal{F}(n-1, d)$. If $n-1 \notin F$, then $F$ is contained in $\operatorname{ast}_{\mathrm{k}_{T}(n)}(n-1)$ and there must be a simplex in $A$ containing $F$ since there is such a simplex for all elements of $\mathrm{lk}_{T}(n)$ by (c). If $n-1 \in F$, then - by (a) - $F \backslash(n-1)$ is not a facet of $\operatorname{lk}_{T}(n)$. Hence there must 
be a simplex $S$ in $\operatorname{ast}_{\mathrm{lk}_{T}(n)}(n-1)$ containing $F \backslash(n-1)$ and therefore the simplex $(S, n-1)$ is in $B$ and contains $F$, which completes the proof.

The proof of (IP) (iii). The simplices in $A$ are pairwise admissible because they are part of $T$, the simplices in $B$ are pairwise admissible because $B$ is a pyramid over a set of admissible simplices. Therefore assume there are $S_{1} \in A$ and $S_{2} \in B$ and a circuit $Z$ with $Z^{+} \subseteq S_{1}$ and $Z^{-} \subseteq S_{2}$, where $n-1 \in S_{2}$ by definition. If $n-1 \notin Z^{-}$then $S_{2}^{\prime}:=S_{2} \backslash(n-1) \cup n$ and $S_{1}$ are not admissible either, contradiction because $S_{1}$ and $S_{2}^{\prime}$ are in $T$. But if we replace $n-1$ by $n$ in $Z$ then we get a circuit $Z^{\prime}$ that again shows that $S_{1}$ and $S_{2}^{\prime}$ are not admissible.

Corollary 4.3. Any triangulation of the cyclic d-polytope $C(n, d)$ with $n$ vertices induces

- a canonical triangulation $\hat{T}$ of $C(n+1, d+1)$ containing $T$ as the link of $n+1$,

- a canonical triangulation $T / n$ of $C(n-1, d-1)$ which is the link of $n$,

- a canonical triangulation $T \backslash n$ of $C(n-1, d)$ containing the antistar of $n$ as a subcomplex, and

- a canonical triangulation $\delta T$ defined as $\hat{T} \backslash n+1$ of $C(n, d+1)$ containing $T$ as a subcomplex.

Remark 4.4. All these constructions - except for the link - are specific for cyclic polytopes and are incorrect for some more general polytopes.

In order to demonstrate that triangulating cyclic polytopes is nevertheless non-trivial, we provide an example showing that they are not greedily triangulable.

Example 4.5. Let $n=8, d=5$ and

$$
\begin{aligned}
& S_{1}:=(3,4,5,6,7,8), \\
& S_{2}:=(1,2,3,6,7,8), \\
& S_{3}:=(1,2,3,4,5,6) .
\end{aligned}
$$

Every pair of these simplices is admissible.

However, consider the facet $F:=(1,3,6,7,8)$ of $S_{2}$ : it is not a facet of $C(8,5)$. Hence, in any triangulation $T$ of $C(8,5)$ that contains $S_{1}, S_{2}$, and $S_{3}$ there must be a simplex $S^{\prime}$ containing $F$. But all three possibilities for such a simplex produce non-admissible pairs. Therefore there is no such triangulation. Hence, one can get stuck by triangulating a cyclic polytope.

\section{THE HIGHER STASHEFF-TAMARI ORDERS}

In this section we describe the notion of increasing bistellar flips (as suggested by EDELMAN \& REINER [6]) in terms of our set-up. This leads to a combinatorial definition of the first higher Stasheff-Tamari order $S_{1}(n, d)$. In contrast to this, the geometric definition of the second higher StasheffTamari order $S_{2}(n, d, t)$ is related to a geometric interpretation $S_{1}(n, d, t)$ of 

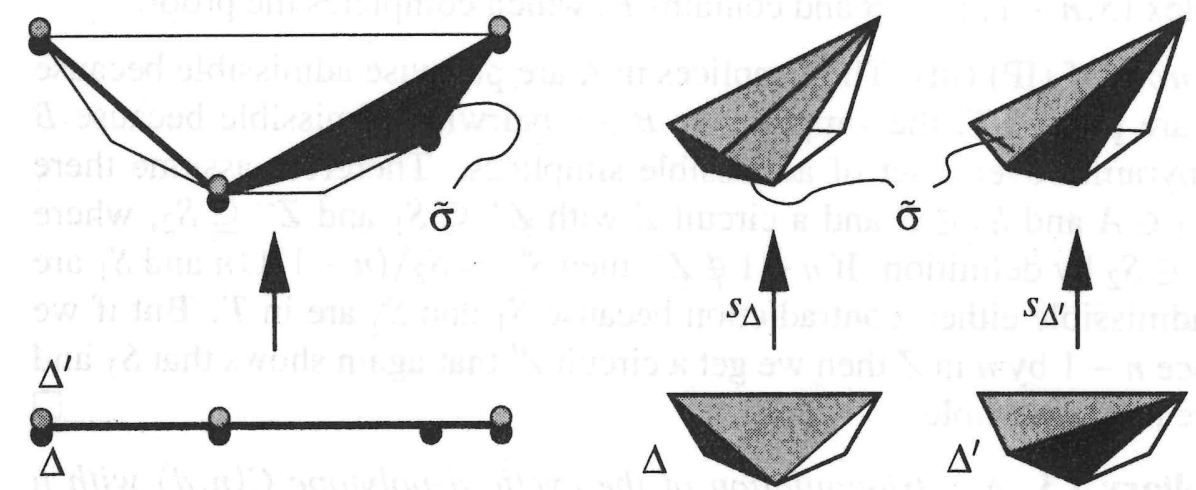

FIGURE 2. Increasing flips in $S_{1}(6,1)$ respectively $S_{1}(5,2)$.

$S_{1}(n, d)$. Specific properties of cyclic polytopes lead to a simple proof of Theorem 1.1.

The set of all triangulations of $C(n, d)$, respectively $C(n, d, t)$, is denoted by $S(n, d)$, respectively $S(n, d, t)$.

Definition 5.1. An increasing (bistellar) flip set in $T \in S(n, d)$ is a simplex $\tilde{S} \in\left(\begin{array}{c}{[n]} \\ d+2\end{array}\right)$ with the property that the set of simplices $\mathcal{F}^{l}(\tilde{S}, d+1)$ is a subset of $T$.

For all $(d+2)$-subsets $\tilde{S}$ of $[n]$ we have the increasing flip function of $\tilde{S}$

$$
\operatorname{flip}_{\tilde{S}}:\left\{\begin{aligned}
S(n, d) & \rightarrow S(n, d), \\
T & \mapsto\left\{\begin{aligned}
& T \backslash \mathcal{F}^{l}(\tilde{S}, d+1) \\
& \cup \mathcal{F}^{u}(\tilde{S}, d+1) \text { if } \mathcal{F}^{l}(\tilde{S}, d+1) \subseteq T \\
& T \text { otherwise. }
\end{aligned}\right.
\end{aligned}\right.
$$

Remark 5.2. By Proposition 3.11 this definition is equivalent to the notion of directed bistellar operations in EDELMAN \& REINER [6].

Remark 5.3 (Geometric Meaning, see Figure 2). Let $t:[n] \rightarrow \mathbb{R}$ be strictly monotone. Let $\Delta$ be a geometric triangulation of $C(n, d, t)$ labelled by $T$, and $\Delta^{\prime}$ geometric triangulation of $C(n, d, t)$ defined by the labels of $\operatorname{flip}_{\tilde{S}}(T)$ for some increasing flip $\tilde{S}$ in $T \in S(n, d)$. Then the geometric lower facets of the $(d+1)$-simplex $\tilde{\sigma}:=v_{d+1} \circ t(\tilde{S})$ in $C(n, d+1, t)$ defined by $\tilde{S}$ are contained in the piecewise linear section $s_{\Delta}$, the geometric upper facets lie in $s_{\Delta^{\prime}}$, and elsewhere the sections coincide.

Definition 5.4. (EDELMAN \& REINER [6]) The first higher Stasheff-Tamari order on $S(n, d)$ is defined via

$$
T_{1} \leq_{1} T_{2} \Longleftrightarrow T_{2}=\operatorname{flip}_{\tilde{S}_{r}} \circ \ldots \circ \text { flip }_{\tilde{S}_{1}}\left(T_{1}\right)
$$

for some sequence $\left(\tilde{S}_{1}, \ldots, \tilde{S}_{r}\right)$ in $\left(\begin{array}{c}{[n]} \\ d+2\end{array}\right)$. The set of all triangulations of $C(n, d)$ with this partial order is denoted by $S_{1}(n, d)$.

The second higher Stasheff-Tamari order on $S(n, d, t)$ is defined via

$$
\Delta_{1} \leq_{2} \Delta_{2} \Longleftrightarrow s_{\Delta}(x)_{d+1} \leq s_{\Delta^{\prime}}(x)_{d+1} \quad \text { for all } x \in C(n, d, t),
$$


that is, $s_{\Delta_{1}}$ lifts $C(n, d)$ weakly lower than $s_{\Delta_{2}}$. It is written as $\mathcal{S}_{2}(n, d, t)$.

Remark 5.5. The triangulation $\mathcal{F}^{l}(n, d+1)$ is locally minimal, the triangulation $\mathcal{F}^{u}(n, d+1)$ is locally maximal in $S_{1}(n, d, t)$.

Moreover, $\mathcal{F}^{l}(n, d+1)$ represents the unique (hence global) minimal element, and $\mathcal{F}^{u}(n, d+1)$ to the unique maximal element of $\mathcal{S}_{2}(n, d, t)$ for all strictly monotone $t:[n] \rightarrow \mathbb{R}$.

EDELMAN \& REINER [6, Conjecture 2.6] conjectured that $S_{1}(n, d)$ is the correct combinatorial model for $\mathcal{S}_{2}(n, d)$, that is, $\mathcal{S}_{2}(n, d, t)$ coincides with $S_{1}(n, d, t)$ for all strictly monotone $t:[n] \rightarrow \mathbb{R}$. Theorem 1.1 shows that, at least the maximal and minimal elements of both partial orders coincide.

In order to prove this, we introduce in the following for all $T$ in $S(n, d)$ a partial order on the set of their simplices. In this context the notion of the parity of "gaps" in linearly ordered sets of Definition 3.2 is again useful.

Definition 5.6. To each $S \in\left(\begin{array}{c}{[n]} \\ d+1\end{array}\right)$ we assign a unique string by

$$
\Gamma:\left\{\begin{aligned}
\left(\begin{array}{c}
{[n]} \\
d+1
\end{array}\right) \rightarrow & \{o, *, e\}^{n} \\
S \mapsto & \left(\gamma_{1}, \ldots, \gamma_{n}\right), \\
& \text { with } \gamma_{i}=\left\{\begin{aligned}
e & \text { if } i \notin S \text { and } \#\{j \in S: j>i\} \text { even, } \\
* & \text { if } i \in S, \\
o & \text { if } i \notin S \text { and } \#\{j \in S: j>i\} \text { odd. }
\end{aligned}\right.
\end{aligned}\right.
$$

(Here the letter " $e$ " denotes an even gap, the letter " $o$ " an odd gap in $S$, while " $*$ " corresponds to an element of $S$.)

Let " $\prec_{(o * e)}$ " be the lexicographic order on $\left(\begin{array}{c}{[n]} \\ d+1\end{array}\right)$ induced by $\Gamma$ and the linear order of letters " $o \prec_{(o * e)} * \prec_{(o * e)} e$."

Definition 5.7. For $S_{1}$ and $S_{2}$ in $T \in S(n, d)$ with $\#\left(S_{1} \cup S_{2}\right)=d+2$ define the relation

$$
S_{1} \prec S_{2} \Longleftrightarrow S_{1} \cap S_{2} \in \mathcal{F}^{u}\left(S_{1}, d\right) \cap \mathcal{F}^{l}\left(S_{2}, d\right) .
$$

Moving from one simplex of a triangulation to an adjacent one can either be considered as moving an element or moving a gap of the support.

Lemma 5.8. Let $T \in S(n, d)$ and $S_{1}, S_{2} \in T$ with $S_{1} \prec S_{2}$. Set $S_{12}:=S_{1} \cap S_{2}$, $S_{1} \backslash S_{12}=: i_{1}$, and $S_{2} \backslash S_{12}=: i_{2}$.

1. If $i_{2}$ is an even gap in $S_{1}$ then $i_{1}$ is an even gap in $S_{2}$ and $i_{1}<i_{2}$, that is, " $\prec$ " moves even gaps to the left.

2. If $i_{2}$ is an odd gap in $S_{1}$ then $i_{1}$ is an odd gap in $S_{2}$ and $i_{1}>i_{2}$, that is, "ঝ" moves odd gaps to the right.

3. A gap changes parity if and only if it lies between $i_{1}$ and $i_{2}$.

Proof. The assumptions imply that $S_{2}$ is obtained from $S_{1}$ by deleting an odd element $i_{1}$ from $S_{1}$ and adding an even gap $i_{2} \notin S_{1}$ to $S_{12}$, or equivalently, the gap $i_{2}$ moves to position $i_{1}$. 
If $i_{1}<i_{2}$ then $i_{2}$ is an even gap in $S_{1}$, and $i_{1}$ is an even gap in $S_{2}$, i. e., the even gap at $i_{2}$ moves to the left. If $i_{2}<i_{1}$ then $i_{2}$ is an odd gap in $S_{1}$, and $i_{1}$ is an odd gap in $S_{2}$, i. e., the odd gap at $i_{2}$ moves to the right.

The third assertion is true because for any label $i \notin\left\{i_{1}, i_{2}\right\}$ not between $i_{1}$ and $i_{2}$ the number of elements to the right stays constant.

Corollary 5.9. The transitive closure of " $\prec$ " is a partial order on the set of all d-simplices in $\left(\begin{array}{c}{[n]} \\ d+1\end{array}\right)$. Ad-simplex $S$ is minimal if and only if all of its lower facets are contained in $\mathcal{F}^{l}(n, d)$; it is maximal if and only if all of its upper facets are in $\mathcal{F}^{u}(n, d)$.

Proof. By Lemma 5.8 we have that

$$
S_{1} \prec S_{2} \Longrightarrow S_{1} \prec(o * e) S_{2} \text {. }
$$

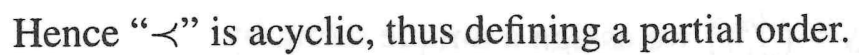

Remark 5.10 (Geometric Meaning). Let $\Delta$ be a triangulation of $C(n, d, t)$. Corollary 5.9 tells us that the repeated transition from one simplex $\sigma \in \Delta$ to an adjacent one docking from below does not create any cycles.

One cannot expect a similar property for triangulations of general polytopes, as is shown by the strongly non-regular triangulation of the twisted capped prism in LEE [12].

Now the following proposition can be proved by combining combinatorial and geometrical facts.

Proposition 5.11. Let $T \in S(n, d) \backslash \mathcal{F}^{u}(n, d+1)$ and $\tilde{T} \in S(n, d+1)$ such that $T$ is a subcomplex of $\tilde{T}$. Then there is a $(d+1)$-simplex $\tilde{S} \in \tilde{T}$ that defines an increasing flip in $T$.

Similarly, for any $T \in S(n, d) \backslash \mathcal{F}^{l}(n, d+1)$ there is a $(d+1)$-simplex that defines a decreasing flip in $T$.

Proof. Choose a simplex $S$ in $T \backslash\left(\mathcal{F}^{u}(n, d+1) \cap T\right)$. Since $S$ is not an upper facet of $C(n, d+1)$ there is an even gap $e$ missing in $S$. Consider $\tilde{S}:=S \cup e$. This $(d+1)$-simplex has the property that one of its lower facets, namely $S$, lies in $T$ by construction.

We now choose a geometric interpretation by fixing $t:[n] \rightarrow \mathbb{R}$, strictly monotone. This gives rise to geometric interpretations $C(n, d, t)$ of $C(n, d)$, $C(n, d+1, t)$ of $C(n, d+1), \tilde{\Delta}$ of $\tilde{T}, \Delta$ of $T$, and $\tilde{\sigma}$ of $\tilde{S}$. Because $T$ is a subcomplex of $\tilde{T}$ we know that its piecewise linear section $s_{\Delta}$ is a subcomplex of $\tilde{\Delta}$. But then $\tilde{\sigma}$ lies weakly above the section $s_{\Delta}$ because at least one of its lower facets, namely $s_{\Delta}(\sigma)$, is contained in $s_{\Delta}$.

If there exists a lower facet $F_{l} \in \mathcal{F}^{l}(\tilde{S}, d+1)$ of $\tilde{S}$ that is not contained in $T$ then either $F_{l}$ is a lower facet of $C(n, d+1)$ - which is impossible because between the geometric interpretation $\sigma^{\prime}$ of $F_{l}$ and the lower facets of $C(n, d+1, t)$ lies the section $s_{\Delta}$ - or there is a simplex $\tilde{S}^{\prime} \in \tilde{T}$ with $F_{l} \subset \tilde{S}^{\prime}$ and $\tilde{S}^{\prime} \prec \tilde{S}$, the geometric interpretation of which is still lying weakly above the section. By continuing this process we will — by Corollary $5.9-$ 

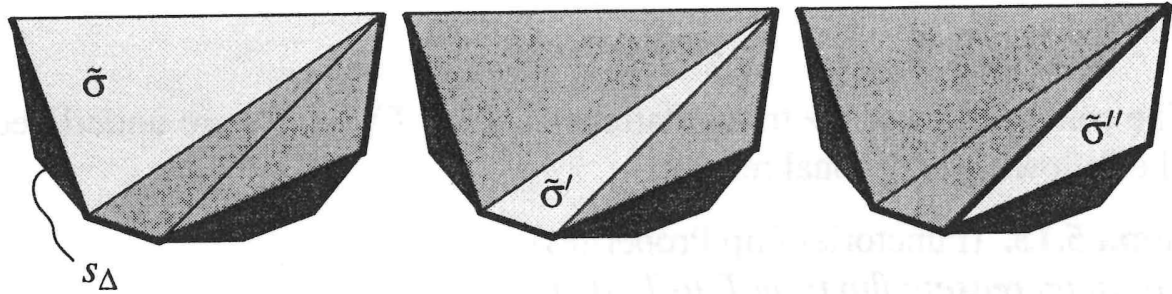

FIGURE 3. Finding an increasing flip in $S_{1}(8,1)$.

end up with a simplex $\tilde{S}^{\prime \prime} \in \tilde{T}$ with $\mathcal{F}^{l}\left(\tilde{S}^{\prime \prime}, d+1\right) \subseteq T$ (see Figure 3 ). The decreasing flip can be found analogously.

We know that all geometric interpretations have the same combinatorial structure, thus the proof is complete.

The special form of the increasing (decreasing) flips in Proposition 5.11 leads to the following result.

Corollary 5.12. Let $\tilde{T}$ be a triangulation of $C(n, d+1)$. Then every linear extension " $\prec_{l}$ " of " $\prec$ " on $\tilde{T}$ defines a maximal chain in $S_{1}(n, d)$ via

$$
\mathcal{F}^{l}(n, d+1)=T_{0} \stackrel{\tilde{S}_{1}}{\leftarrow} T_{1} \stackrel{\tilde{S}_{2}}{\leftarrow} \ldots \stackrel{\tilde{S}_{r-1}}{\leftarrow} T_{r-1} \stackrel{\tilde{S}_{r}}{\leftarrow} T_{r}=\mathcal{F}^{u}(n, d+1),
$$

where

$$
\tilde{T}=\left\{\tilde{S}_{1}, \tilde{S}_{2}, \ldots, \tilde{S}_{r}\right\}, \quad \tilde{S}_{1} \prec_{l} \tilde{S}_{2} \prec_{l} \ldots \prec_{l} \tilde{S}_{r} .
$$

Proof of Theorem 1.1. In order to prove (i) we show that any triangulation of $C(n, d)$ is on a chain from $\mathcal{F}^{l}(n, d+1)$ to $\mathcal{F}^{u}(n, d+1)$. Let $T$ be an arbitrary triangulation of $C(n, d)$. Then, by Theorem $4.3, \delta T$ is a triangulation of $C(n, d+1)$ containing $T$ as a subcomplex. Thus, by Proposition 5.11 and induction, we can connect $T$ to $\mathcal{F}^{u}(n, d+1)$ by a sequence of increasing flips (compare Figure 3), and to $\mathcal{F}^{l}(n, d+1)$ by a sequence of decreasing flips, which implies the assertion.

For the proof of (ii) observe that, by the definition of increasing bistellar flips, any chain

$$
c: \mathcal{F}^{l}(n, d+1) \stackrel{\tilde{S}_{1}}{<} \cdots \stackrel{\tilde{S}_{r}}{<} \mathcal{F}^{u}(n, d+1)
$$

from $\mathcal{F}^{l}(n, d+1)$ to $\mathcal{F}^{u}(n, d+1)$ defines a triangulation $T_{c}$ of $C(n, d+1)$ via

$$
T_{c}:=\left\{\tilde{S}_{1}, \ldots, \tilde{S}_{r}\right\}
$$

hence factoring out the order of $c$. For the converse, let $\tilde{T}$ be an arbitrary triangulation of $C(n, d+1)$. Then, by Corollary 5.12,

$$
c_{\tilde{T}}:=T_{\prec(\rho * e)}
$$

is a chain in $\mathcal{S}_{1}(n, d)$ from $\mathcal{F}^{l}(n, d+1)$ to $\mathcal{F}^{u}(n, d+1)$.

Part (iii) follows directly from Corollary 5.12. 
The central roles of the triangulations $\hat{T}, T / n, T \backslash n, \delta(T)$ are underlined by the following additional results.

Lemma 5.13. (Functorial Flip Properties) If $\tilde{S}$ is an increasing flip from $T$ to $T^{\prime}$ then

$$
(\tilde{S})_{\prec_{l}}:=\left\{\tilde{S} \backslash \tilde{s}_{d+2} \cup\{j, j+1\}: \tilde{s}_{d+1}<j<\tilde{s}_{d+2}\right\}_{\prec_{l}}
$$

is a decreasing flip sequence from $\hat{T}$ to $\hat{T}^{\prime}$,

$$
(\tilde{S} / n):=\left\{\begin{aligned}
(\tilde{S} \backslash\{n\}) & \text { if } n \in \tilde{S}, \\
() & \text { otherwise, }
\end{aligned}\right.
$$

is an increasing flip from $T / n$ to $T^{\prime} / n$,

$$
(\tilde{S} \backslash n):=\left\{\begin{aligned}
(\tilde{S}) & \text { if } n \notin \tilde{S}, \\
(\tilde{S} \backslash\{n\} \cup\{n-1\}) & \text { if } n \in \tilde{S}, n-1 \notin \tilde{S}, \\
() & \text { otherwise, }
\end{aligned}\right.
$$

is a decreasing flip sequence from $T \backslash n$ to $T^{\prime} \backslash n$, where " $\prec_{l}$ " is any linear extension of " $\prec$."

Proposition 5.14. (Functorial Order Properties)

(i) The map

$$
\hat{\imath}\left\{\begin{aligned}
S_{1}(n, d) & \rightarrow S_{1}(n+1, d+1), \\
T & \mapsto \hat{T}
\end{aligned}\right.
$$

is order-reversing.

(ii) The map

$$
\cdot / n:\left\{\begin{aligned}
S_{1}(n, d) & \rightarrow S_{1}(n-1, d-1) \\
T & \mapsto T / n
\end{aligned}\right.
$$

is order-reversing.

(iii) The map

$$
\cdot \backslash n:\left\{\begin{aligned}
\mathcal{S}_{1}(n, d) & \rightarrow \mathcal{S}_{1}(n-1, d), \\
T & \mapsto T \backslash n,
\end{aligned}\right.
$$

is order-preserving.

(iv) The map

$$
\delta:\left\{\begin{aligned}
\mathcal{S}_{1}(n, d) & \rightarrow \mathcal{S}_{1}(n, d+1), \\
T & \mapsto \delta(T),
\end{aligned}\right.
$$

is order-reversing.

Corollary 5.15. Every chain in $S_{1}(n, d)$ corresponding to a flip sequence $(\tilde{T}):=\left(\tilde{S}_{1}, \ldots, \tilde{S}_{r}\right)$ gives rise to flip sequences

(i) $(\tilde{T})_{\prec_{l}}$ in $S_{1}(n+1, d+1)$,

(ii) $(\tilde{T} / n)_{\prec_{l}}$ in $S_{1}(n-1, d-1)$,

(iii) $(\tilde{T} \backslash n)_{\prec}$ in $S_{1}(n-1, d)$, and

(iv) $\delta(\tilde{T})_{\prec l}$ in $S_{1}(n, d+1)$. 


\section{HIGHER BRUHAT ORDERS}

In this section we recall the basic definitions and theorems in the framework of higher Bruhat orders and answer a question of ZIEGLER [15]. Let $\mathcal{L}$ is a linearly ordered finite set. The reader may consider $\mathcal{L}$ as the set $[n]$, without loss of generality.

Definition 6.1. (MANIN \& SCHECHTMAN [13], ZIEGLER [15])

- For some $(k+1)$-subset $P:=\left(p_{1}, \ldots, p_{k+1}\right)$ of $\mathcal{L}$ the set of its $k$ subsets

$$
\mathcal{P}=\left(\begin{array}{l}
P \\
k
\end{array}\right)=\left\{P \backslash p_{v}: v=1, \ldots, k+1\right\}
$$

is a k-packet of $\mathcal{L}$. It is naturally ordered by $P \backslash p_{\nu}<P \backslash p_{\mu} \Longleftrightarrow \mu<v$, the lexicographic order.

- An ordering $\alpha$ of $\left(\begin{array}{l}\mathcal{L} \\ k\end{array}\right)$ is admissible if the elements of any $(k+1)$ packet appear in lexicographic or in reverse-lexicographic order. Two orderings $\alpha$ and $\alpha^{\prime}$ are equivalent if they differ by a sequence of interchanges of two neighbors that do not lie in a common packet.

- The inversion set $\operatorname{inv}(\alpha)$ of an admissible ordering $\alpha$ is the set of all $(k+1)$-subsets of $\mathcal{L}$ the $k$-subsets of which appear in reverse-lexicographic order in $\alpha$.

- A set $U$ of $(k+1)$-subsets of $\mathcal{L}$ is consistent if its intersection with any $(k+1)$-packet $\mathcal{P}$ of $\mathcal{L}$ is a beginning or an ending segment of $\mathcal{P}$ with respect to the lexicographic order on $\mathcal{P}$.

- The set of all equivalence classes of admissible orders of $\left(\begin{array}{l}\mathfrak{L} \\ k\end{array}\right)$ partially ordered by single-step-inclusion of inversion sets - that is, $[\alpha] \leq\left[\alpha^{\prime}\right]$ if and only if

$$
\operatorname{inv}(\alpha)=U_{1} \subset U_{2} \subset \cdots \subset U_{K}=\operatorname{inv}\left(\alpha^{\prime}\right)
$$

with $\# U_{v} \backslash U_{v-1}=1$ and all $U_{v}$ are admissible - is the higher Bruhat order $\mathcal{B}(\mathcal{L}, k)$, where $\mathcal{B}(n, k)$ denotes $\mathcal{B}([n], k)$.

- For an inversion set $U \in \mathcal{B}(\mathcal{L}, k)$ define

$$
\partial U:=\left\{I \in\left(\begin{array}{c}
\mathcal{L} \\
k+2
\end{array}\right): I \backslash i_{1} \notin U, I \backslash i_{k+2} \in U\right\} .
$$

The structure of $\mathcal{B}(\mathcal{L}, k)$ does of course only depend on the cardinality of $\mathcal{L}$, but the general setting leads to some advantages in the notation of functorial constructions. For simplicity, however, we switch now to $\mathcal{B}(n, k)$.

Theorem 6.2. (MANIN \& SCHECHTMAN [13], ZIEGLER [15]) The higher Bruhat order $\mathcal{B}(n, k)$ is a ranked poset with rank function $r(U)=\# U$. Moreover, it has a unique minimal element $\hat{0}_{n, k}=\varnothing$ and a unique maximal element $\hat{1}_{n, k}=\left(\begin{array}{c}{[n]} \\ k+1\end{array}\right)$.

The following Theorem gives a more geometric insight into the structure of higher Bruhat orders.

Theorem 6.3. (ZIEGLER[15]) The higher Bruhat order $\mathcal{B}(n, k)$ is isomorphic to 
1. the set of all consistent sets $U$ of $(k+1)$-subsets of $[n]$ with singlestep-inclusion-order,

2. the set of (equivalence classes of) extensions of the cyclic hyperplane arrangement $X^{n, n-k-1}$ by a new pseudo-hyperplane in general position, partially ordered by single-step-inclusion of the sets of vertices on "the negative side,"

3. the set of maximal chains of inversion sets in $\mathcal{B}(n, k-1)-$ corresponding to orders of $k$-sets - modulo equivalence of admissible orders.

The following notations for deletion and contraction in $\mathcal{B}(n, k)$ provide intuition via the corresponding notions in $X^{n, n-k-1}$.

Definition 6.4. For $U \in \mathcal{B}(n, k)$ define

$$
\begin{aligned}
& U / n:=\{I \backslash n: n \in I, I \in U\}, \\
& U \backslash n:=\{I \in U: n \notin I\} .
\end{aligned}
$$

In order to construct inversion sets in $\mathcal{B}(n+1, k+1)$ from inversion sets in $\mathcal{B}(n, k)$ and in $\mathcal{B}(n, k+1)$ the following Theorem is useful.

Theorem 6.5. (ZIEGLER [15]) Let $U$ be an inversion set in $\mathcal{B}(n, k)$ and $V$ be an inversion set in $\mathcal{B}(n, k+1)$. Then $U^{\prime}:=V \cup U *(n+1)$ is consistent if and only if

$$
\partial U \subseteq V \text { and } \partial \complement U \subseteq \complement V .
$$

Corollary 6.6. The following maps from $\mathcal{B}(n, k)$ to $\mathcal{B}(n+1, k+1)$ are injective:

$$
\begin{array}{rlrl}
U & \mapsto \widetilde{U}:=U *(n+1) \cup \partial U, & & \text { (extension) } \\
U \mapsto \hat{U}:=U *(n+1) \cup \delta(U)=U *(n+1) \cup(U \backslash n), & & \text { (expansion) }
\end{array}
$$

where $\delta(U)$ is defined as

$$
\delta(U):=\left\{I \in\left(\begin{array}{c}
{[n]} \\
k+2
\end{array}\right): I \backslash i_{k+2} \in U\right\} .
$$

The extension is not order-preserving in general. But the following definition yields a canonical single-step-inclusion order for the expansion of $U$ from an arbitrary single-step-inclusion order of $U$.

Definition 6.7. For some $U \in \mathcal{B}(n, k)$ with a given single-step-inclusionorder $\Omega(U)=\left(\Omega\left(U^{\prime}\right), I\right)$ define the following order $\hat{\Omega}$ : For $n=k+1$ start with

$$
\hat{\Omega}(\{[n]\}):=([n+1])
$$

corresponding to $\Omega(\{[n]\})=([n])$ in $\mathcal{B}(n, k)$. If $n>k+1$ and $\hat{\Omega}\left(\hat{U}^{\prime}\right)$ is already constructed then define

$$
\hat{\Omega}(\hat{U}):=\left(\hat{\Omega}\left(\hat{U}^{\prime}\right), \hat{\Omega}(\partial I), I \cup\{n+1\}, \hat{\Omega}(\delta I \backslash \partial I)\right),
$$


where the orders on $\partial I$ and $\delta I \backslash \partial I$ are given recursively by restriction of $\hat{\Omega}\left((U \backslash n)^{\uparrow}\right)$.

Proposition 6.8. For all $U \in \mathcal{B}(n, k)$ and all single-step-inclusion orders $\Omega$ of $U$ the order $\hat{\Omega}$ is a single-step-inclusion order of the expansion $\hat{U}$ of $U$ in $\mathcal{B}(n+1, k)$.

Proof. The following properties make sure that no cycles are produced:

$$
\begin{aligned}
& \delta(U) \backslash n=\delta(U \backslash n), \\
& \partial(U) \backslash n=\partial(U \backslash n) .
\end{aligned}
$$

At each single-step-inclusion step all packets in $\mathcal{B}(n, k+1)$ are consistent by induction. From the remaining packets only those containing $I \cup\{n+1\}$ are involved.

If $n \notin I$ then the order increases just by $I \cup\{n\}$ which is consistent because $\Omega$ is a single-step-inclusion order of $U$ and $\hat{U}^{\prime}$ is already ordered consistently.

Let $n$ be in $I$. For all packets $\mathcal{P}$ containing $I \cup\{n+1\}$ either $\mathcal{P} / n+1$ is completely contained in $U$ or only $I$ meets $U$. In the first case the only element $P \backslash a^{\prime}$ of $\mathcal{P} \backslash n+1$ comes before $I \cup\{n+1\}$ in $\hat{\Omega}$, in the second case $I \cup\{n+1\}$ is positioned after $P \backslash n+1$ in $\hat{\Omega}$; both cases lead to consistent orders on $\mathcal{P}$.

From this we derive the promised result.

Theorem 6.9. The expansion

$$
\hat{\imath}\left\{\begin{aligned}
\mathcal{B}(n, k) & \rightarrow \mathcal{B}(n+1, k+1), \\
U & \mapsto \hat{U},
\end{aligned}\right.
$$

is an order-preserving embedding that maps $\widehat{0}_{n, k}$ to $\widehat{0}_{n+1, k+1}$ and $\widehat{1}_{n, k}$ to $\widehat{1}_{n+1, k+1}$.

\section{THE CONNECTION BETWEeN $\mathcal{B}(n-2, d-1)$ AND $\mathcal{S}_{1}(n, d)$}

In this section we present an order-preserving map from the higher Bruhat order $\mathcal{B}(] n[, d-1) \cong \mathcal{B}(n-2, d-1)$ to the poset $\mathcal{S}_{1}(n, d)$ of all triangulations of $C(n, d)$. This map is obtained by two different constructions, each of them providing complementary parts of the properties claimed. It is not quite clear whether this map coincides with the map suggested by Kapranov \& Voevodsky [11].

We start with some additional specific properties of triangulations of cyclic polytopes.

Lemma 7.1. Let $T \in S_{1}(n, d)$. Then for each $(d-1)$-subset $\left(s_{2}, \ldots, s_{d}\right)$ there is at most one simplex $S \in T$ with $S=\left(s_{1}, s_{2}, \ldots, s_{d}, s_{d+1}\right)$ for some $s_{1}<s_{2}$ and some $s_{d+1}>s_{d}$. 
Proof. Assume there were $S \neq S^{\prime} \in T$ and

$$
\begin{aligned}
S & =\left(s_{1}, s_{2}, \ldots, s_{d}, s_{d+1}\right), \\
S^{\prime} & =\left(s_{1}^{\prime}, s_{2}, \ldots, s_{d}, s_{d+1}^{\prime}\right) .
\end{aligned}
$$

Either $s_{1} \neq s_{1}^{\prime}$ or $s_{d+1} \neq s_{d+1}^{\prime}$. If $s_{1}<s_{1}^{\prime}$ then define

$$
Z:= \begin{cases}\left(s_{1}, s_{1}^{\prime}, \ldots, s_{d}, s_{d+1}^{\prime}\right) & \text { if } d \text { even } \\ \left(s_{1}, s_{1}^{\prime}, \ldots, s_{d}, s_{d+1}\right) & \text { if } d \text { odd. }\end{cases}
$$

In any case $Z^{+} \subseteq S$ and $Z^{-} \subseteq S^{\prime}$.

The cases $s_{1}>s_{1}^{\prime}, s_{d+1}<s_{d+1}^{\prime}$, and $s_{d+1}>s_{d+1}^{\prime}$ are analogous.

Definition 7.2. For $S:=\left(s_{1}, \ldots, s_{d+1}\right) \in T \in S_{1}(n, d)$ let $X_{S}:=\left(s_{2}, \ldots, s_{d}\right)$ be the central set of $S$. The number $l_{S}:=s_{1}$ is called the left boundary, the number $r_{S}:=s_{d+1}$ the right boundary of $X_{S}$ in $T$.

Corollary 7.3. Any triangulation $T$ of $C(n, d)$ is determined by its set of central sets and their boundaries.

Lemma 7.4. In every triangulation $T$ of $C(n, d)$ every interval of length $(d-1)$ in $[2, n-1]$ appears as a central set of some simplex $S \in T$.

Proof. Here is a proof for $d$ odd: Let $T$ be in $S_{1}(n, d)$ and $I$ an interval of length $d-1$. From Gale's evenness criterion it follows that $I$ is contained in exactly two facets of $C(n, d)$, namely $(1, I)$ and $(I, n)$. Therefore, there must be a simplex $S_{1}$ in the triangulation $T$ containing $(1, I)$.

If $S_{1}=(1, I, r)$ we are done. Otherwise $S_{1}=\left(1, l_{1}, I\right)$. Because $\left(l_{1}, I\right)$ is not a facet of $C(n, d)$ there must be another simplex $S_{2} \in T$ with $\left(l_{1}, I\right) \subseteq S_{2}$. If $S_{2}=\left(l_{1}, I, r\right)$ we are done. Otherwise proceed as above. Because of Lemma 7.1 at each step $l_{i}<l_{i+1}$. Hence there must be a $k$ and an $r$ such that the simplex $S_{k}=\left(l_{k-1}, I, r\right)$ is in $T$.

The case $d$ even is analogous where the corresponding facets of $C(n, d)$ are $\left(i_{1}-1, I\right)$ and $\left(I, i_{d}+1\right)$ and the sequence of the $l_{k}$ is decreasing.

We start now to construct a map by defining a natural family of functions on $S_{1}(n, d)$.

Definition 7.5. For an element $I=\left(i_{1}, \ldots, i_{d}\right) \in\left(\begin{array}{c}n[n \\ d\end{array}\right)$ define the map

$$
\text { flip }_{I}:\left\{\begin{aligned}
S_{1}(n, d) & \rightarrow S_{1}(n, d), \\
T & \mapsto\left\{\begin{aligned}
\operatorname{flip}_{(l, I, r)}(T) & \text { if }(l, I, r) \text { is an increasing flip, } \\
T & \text { otherwise. }
\end{aligned}\right.
\end{aligned}\right.
$$

For an inversion set $U \in \mathcal{B}(] n[, d-1)$ let $\left(I_{i}\right)_{i=1, \ldots, \# U}$ be a single-stepinclusion-order of the elements of $U$, i. e., $\bigcup_{i=1}^{K} I_{i}$ is consistent for all $K=$ $1, \ldots, \# U$. The flip-map $\mathcal{T}_{\text {flip }}$ is now defined as follows:

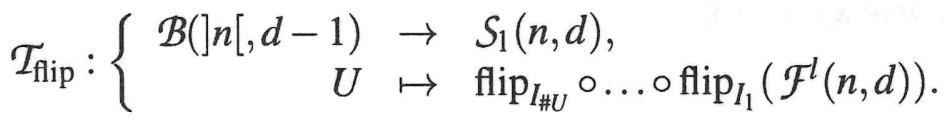


Remark 7.6. At this point it is not obvious that this definition is independent of the special order $\left(I_{i}\right)_{i=1, \ldots, \# U}$. But in any case $\mathcal{T}_{\text {flip }}$ maps each $U \in \mathcal{B}(] n[, d-1)$ to a triangulation in $S_{1}(n, d)$ and it is obviously orderpreserving.

Definition 7.7. For $i \in I \in\left(\begin{array}{c}]^{n}[ \\ d\end{array}\right)$ define the index of $i$ in $I$ as

$$
\operatorname{ind}_{I}(i):=k \quad \text { if } I=\left(i_{1}, \ldots, i=i_{k}, \ldots, i_{d}\right) \text {. }
$$

Definition 7.8. For an inversion set $U \in \mathcal{B}(] n[, d-1)$ define the central set of $U$ as

$$
\begin{aligned}
& X_{U}:=\left\{X=\left(x_{1}, \ldots, x_{d-1}\right) \in\left(\begin{array}{l}
\ln [1 \\
d-1
\end{array}\right): X \cup j \in U \quad \forall j \in[n] \backslash X:\right. \\
& x_{1}<j<x_{d-1}, \\
& d-\operatorname{ind}_{X \cup j}(j) \text { even, } \\
& X \cup j \notin U \quad \forall j \in[n] \backslash X: \\
& x_{1}<j<x_{d-1}, \\
&\left.d-\text { ind }_{X \cup j}(j) \text { odd }\right\} .
\end{aligned}
$$

Definition 7.9. For an inversion set $U \in \mathcal{B}(] n[, d-1)$ define the left boundary function of $U$ as

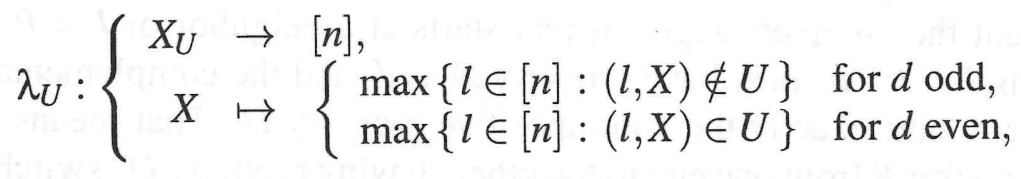

and the right boundary function of $U$ as

$$
\rho_{U}:\left\{\begin{aligned}
X_{U} & \rightarrow[n] \\
X & \mapsto \min \{r \in[n]:(X, r) \notin U\}
\end{aligned}\right.
$$

with the additional notation

$$
\min (\varnothing):=n \text { and } \max (\varnothing):=1 \text {. }
$$

Definition 7.10. Now define the direct map $\mathcal{T}_{\text {dir }}$ as

$$
\mathcal{T}_{\text {dir }}:\left\{\begin{aligned}
\mathcal{B}(] n[, d-1) & \rightarrow S_{1}(n, d), \\
U & \mapsto\left\{\left(\lambda_{U}(X), X, \rho_{U}(X)\right): X \in X_{U}\right\} .
\end{aligned}\right.
$$

Remark 7.11. Here it is neither obvious that $\mathcal{T}_{\text {dir }}(U)$ is indeed a triangulation nor that the map is order-preserving, but it is uniquely defined.

Proposition 7.12. Let $U$ and $U^{\prime}:=U \cup\{I\}$ be inversion sets in $\mathcal{B}(] n[, d-$ 1). Define the following two properties for some $i_{k} \in I, 1<k<d-1$.

Property $A: I \backslash i_{k} \in X_{U}$ but $I \backslash i_{k} \notin X_{U^{\prime}}$,

Property $B: I \backslash i_{k} \notin X_{U}$ but $I \backslash i_{k} \in X_{U^{\prime}}$.

Then the following hold:

(i) If $i_{k}$ has Property $A$ then all $i_{m} \in I$ with $m \equiv k \bmod 2$ have Property $A$ as well,

(ii) If $i_{k}$ has Property $B$ then all $i_{m} \in I$ with $m \equiv k \bmod 2$ have Property $B$ as well. 
Proof. From Definition 7.8 we know that

- $i_{k}$ has Property $A$ if and only if $d-k$ is odd and $i_{k}$ has Property $C$, namely

- ind $\operatorname{in}_{I \backslash i_{k} \cup j}(j)$ is even for all $j \notin I$ with $i_{1}<j<i_{d-1}$ and $I \backslash i_{k} \cup j \in U$, and

- $\operatorname{ind}_{I \backslash i_{k} \cup j}(j)$ is odd for all $j \notin I$ with $i_{1}<j<i_{d-1}$ and $I \backslash i_{k} \cup j \notin U$,

- $i_{k}$ has Property $B$ if and only if $d-k$ is even and $i_{k}$ has Property $C$.

In the sequel we will show that Property $C$ for $i_{k}$ induces Property $C$ for all $i_{m} \in I$.

Assume $i_{k} \in I$ has Property $C$. Let $j \notin I, i_{1}<j<i_{d}$ be arbitrary. (If there is no such $j$ we are done.) Consider the inversion $J:=(I \cup j) \backslash i_{k}$. From Property $C$ we know that $J$ has Property $D$, namely

$$
J \subseteq \begin{cases}U & \text { if } \operatorname{ind}_{J}(j) \text { even } \\ \bar{U} & \text { if } \operatorname{ind}_{J}(j) \text { odd }\end{cases}
$$

Now we investigate the $d$-packet $P:=I \cup J$. Because both $U$ and $U^{\prime}$ are consistent the complete segment that starts at a neighbor of $I=P \backslash j$ and contains $J=P \backslash i_{k}$ must have property $D$ as $J$, and the complementary segment must have exactly the contrapositive property $\bar{D}$. That means by parsing the packet $P$ from one end to the other "having property $D$ " switches at $I=P \backslash j$.

In other words, $I \backslash i_{m} \cup j \in U$ if and only if $I \backslash i_{k} \cup j \in U$ for all $i_{m}$ lying on the same side of $j$ as $i_{k}$ in $P$ and $I \backslash i_{m} \cup j \in U$ if and only if $I \backslash i_{k} \cup j \notin U$ for all $i_{m}$ lying on the opposite side of $j$ as $i_{k}$.

Additionally, if $m$ is congruent $k$ modulo 2 then $\operatorname{ind}_{I \backslash i_{m} \cup j}(j)$ is congruent $\operatorname{ind}_{I \backslash i_{k} \cup j}(j)$ modulo 2 if and only if $i_{m}$ lies on the same side of $j$ as $i_{k}$ in $P$, but - since $j$ was arbitrary - this means that $i_{m}$ has Property $C$.

Remark 7.13. The above Proposition roughly states that for $I \backslash i_{m}$ "being contained in the central set of $U$ " for all possible $m$ only depends on whether $I$ is in $U-$ not on whether some inversion $I \backslash i_{m} \cup j$ is in $U-$ whenever this is correct for one $m$.

Proposition 7.14. Let $U$ and $U^{\prime}$ as above. Then the following hold for all $1<l<i_{1}$ and $i_{d-1}<r<n$ :

$$
\begin{aligned}
& \left(l, I \backslash i_{k}\right) \in U \Longleftrightarrow\left(l, I \backslash i_{m}\right) \in U \quad \text { for all } m \equiv k \bmod 2, \\
& \left(I \backslash i_{k}, r\right) \in U \Longleftrightarrow\left(I \backslash i_{m}, r\right) \in U \quad \text { for all } m \equiv k \bmod 2 .
\end{aligned}
$$

Proof. The proof is analogous to the proof of Proposition 7.12 with $j$ replaced by $l, r$.

Theorem 7.15. The maps $\mathcal{T}_{\text {flip }}$ and $\mathcal{T}_{\text {dir }}$ coincide.

Proof. We will show that $\mathcal{T}_{\text {flip }}(U)=\mathcal{T}_{\text {dir }}(U)$ for all $U \in \mathcal{B}(] n[, d-1)$. Because $\mathcal{B}(] n[, d-1)$ has a unique minimal element $\varnothing$ we can proceed by induction on $\# U$. 
The proof for $U=\varnothing$ is a simple computation. Therefore we assume that the claim is true for some inversion set $U$ and we will show that then the claim is also true for all consistent $U^{\prime}:=U \cup\{I\}$.

The following points are to check:

1. If $\mathcal{T}_{\text {dir }}\left(U^{\prime}\right) \neq \mathcal{T}_{\text {dir }}(U)$ then there exist $1 \leq l<i_{1}$ and $i_{d}<r \leq n$ such that $(l, I, r)$ is an increasing flip in $\mathcal{T}_{\text {flip }}(U)=\mathcal{T}_{\text {dir }}(U)$, and

2. if the $(d+2)$-set $(l, I, r)$ is an increasing flip in $\mathcal{T}_{\text {flip }}(U)=\mathcal{T}_{\text {dir }}(U)$ then $\mathcal{T}_{\text {dir }}\left(U^{\prime}\right)=\operatorname{flip}_{I} \mathcal{I}_{\text {dir }}(U)$.

From Proposition 7.12 it follows that the assertions 1 and 2 are correct as far as the central sets of $U$ or $U^{\prime}$, resp., are concerned.

From Proposition 7.14 and the corresponding definitions in 7.9 we get that in the situations of both 1 and 2 the left and right boundary functions are constant on the sets $I \backslash i_{k}$ with $1<k<d-1$, i. e., there exist $l$ and $r$ with $1<l<i_{1}$ and $i_{d-1}<r<n$ such that

$$
\lambda_{U}\left(I \backslash i_{k}\right)=l, \quad \rho_{U}\left(I \backslash i_{k}\right)=r .
$$

Moreover, it follows that

$$
\begin{aligned}
& \lambda_{U}\left(I \backslash i_{1}\right)=\left\{\begin{array}{cl}
i_{1} & \text { for } d \text { odd } \\
l & \text { for } d \text { even, }
\end{array}\right. \\
& \lambda_{U}\left(I \backslash i_{d-1}\right)=l, \\
& \rho_{U}\left(I \backslash i_{1}\right)=r, \\
& \rho_{U}\left(I \backslash i_{d-1}\right)=i_{d-1} .
\end{aligned}
$$

After having added $I$ to the inversion set $U$ we have

$$
\begin{aligned}
& \lambda_{U^{\prime}}\left(I \backslash i_{k}\right)=\left\{\begin{aligned}
l & \text { for } d \text { odd, } \\
i_{1} & \text { for } d \text { even, }
\end{aligned}\right. \\
& \rho_{U^{\prime}}\left(I \backslash i_{k}\right)=r . \\
& \lambda_{U^{\prime}}\left(I \backslash i_{1}\right)=l, \\
& \rho_{U^{\prime}}\left(I \backslash i_{1}\right)=r, \\
& \lambda_{U^{\prime}}\left(I \backslash i_{d-1}\right)=l, \\
& \rho_{U^{\prime}}\left(I \backslash i_{d-1}\right)=r .
\end{aligned}
$$

With this the proof of Theorem 7.15 is complete.

Corollary 7.16. The map

$$
\mathcal{T}:=\mathcal{T}_{\text {flip }}=\mathcal{T}_{\text {dir }}
$$

is well-defined and order-preserving.

We finish the paper by stating - as a bonus track without a proof - the following connections between the constructions of this paper.

Proposition 7.17. (Functorial Relations)

$$
\begin{aligned}
\mathcal{T}(\hat{U}) & =(\mathcal{T}(U)), \\
\mathcal{T}(U \backslash n-1) & =\mathcal{T}(U) \backslash n, \\
\mathcal{T}(\delta U) & =\delta \mathcal{T}(U) .
\end{aligned}
$$

The analogous property for the link does not hold in general! 


\section{ACKNOWLEDGEMENTS}

I would like to thank Jesús Antonio de Loera and Victor Reiner for their helpful comments and corrections, and especially my advisor Günter M. Ziegler for his patience and support, and many fruitful discussions.

\section{REFERENCES}

1. Louis J. Billera, Paul Filliman, and Bernd Sturmfels, Constructions an complexity of secondary polytopes, Advances in Mathematics 83 (1990), 155-179.

2. Louis J. Billera, Israel M. Gelfand, and Bernd Sturmfels, Duality and minors of secondary polyhedra, Journal of Combinatorial Theory Ser. B, 57 (1993), 258-268.

3. Louis J. Billera, Mikhail M. Kapranov, and Bernd Sturmfels, Cellular strings on polytopes, Proceedings of the American Mathematical Society 122 (1994), 549-555.

4. Anders Björner, Michel Las Vergnas, Bernd Sturmfels, Neil White, and Günter M. Ziegler, Oriented matroids, Encyclopedia of Mathematics, vol. 46, Cambridge University Press, Cambridge, 1993.

5. Jesús de Loera, Triangulations of polytopes and computational algebra, Ph.D. thesis, Cornell University, 1995.

6. Paul H. Edelman and Victor Reiner, The higher Stasheff-Tamari posets, Preprint July 1995, Mathematika, to appear.

7. Herbert Edelsbrunner, Algorithms in Geometry and Topology, Course script 497, University of Illinois, Urbana-Champaign, Spring 1993.

8. Izrail M. Gelfand, Mikhail M. Kapranov, and Andrei V. Zelevinsky, Discriminants of polynomials in several variables and triangulations of Newton polyhedra, Leningrad Mathematical Journal 2 (1991), 449-505.

9. Branko Grünbaum, Convex Polytopes, Interscience, London, 1967, Revised edition (V. Klee and P. Kleinschmidt, eds.), Graduate Texts in Mathematics, Springer-Verlag, in preparation.

10. Barry Joe, Three dimensional triangulations from local transformations, SIAM Journal of Scientific Statistical Computation 10 (1989), 718-741.

11. Mikhail M. Kapranov and V. A. Voevodsky, Combinatorial-geometric aspects of polycategory theory: pasting schemes and higher Bruhat orders (list of results), Cahiers de Topologie et Géométrie différentielle catégoriques 32 (1991), no. 1, 11-27.

12. Carl Lee, Triangulations of polytopes, Preprint, July 1995; CRC Handbook of Discrete and Computational Geometry (J. E. Goodman, J. O'Rourke, eds.), to appear.

13. Yu. I. Manin and V. V. Schechtman, Arrangements of hyperplanes, higher braid groups and higher Bruhat orders, Advanced Studies in Pure Mathematics 17 (1989), 289-308.

14. Jörg Rambau and Günter M. Ziegler, Projections of polytopes and the Generalized Baues Conjecture, Preprint TU-Berlin No. 429/1995, Discrete \& Computational Geometry, to appear.

15. Günter M. Ziegler, Higher Bruhat orders and cyclic hyperplane arrangements, Topology 32 (1993), no. 2, 259-279. 
Reports from the group

\section{"Algorithmic Discrete Mathematics" \\ of the Department of Mathematics, TU Berlin}

496/1996: Jörg Rambau: Triangulations of Cyclic Polytopes and higher Bruhat Orders

483/1995: Rolf H. Möhring and Markus W. Schäffter: Approximation Algorithms for Scheduling Series-Parallel Orders Subject to Unit Time Communication Delays

478/1995: Sven G. Bartels: The complexity of Yamnitsky and Levin's simplices algorithm

477/1995: Jens Gustedt, Michel Morvan and Laurent Viennot: A compact data structure and parallel algorithms for permutation graphs, to appear in : Nagl et al., editors, Graph-Theoretic Concepts in Computer Science, Proccedings of the 20th International Workshop WG '95.

476/1995: Jens Gustedt: Efficient Union-Find for Planar Graphs and other Sparse Graph Classes

475/1995: Ross McConnell and Jeremy Spinrad: Modular decomposition and transitive orientation

474/1995: Andreas S. Schulz: Scheduling to Minimize Total Weighted Completion Time: Performance Guarantees of LP-Based Heuristics and Lower Bounds

473/1995: Günter M. Ziegler: Shelling Polyhedral 3-Balls and 4Polytopes

472/1995: Martin Henk, Jürgen Richter-Gebert and Günter M. Ziegler: Basic Properties of Convex Polytopes

471/1995: Jürgen Richter-Gebert and Günter M. Ziegler: Oriented Matroids

465/1995: Ulrich Betke and Martin Henk: Finite Packings of Spheres

463/1995: Ulrich Hund: Every simplicial polytope with at most $d+4$ vertices is a quotient of a neighborly polytope

462/1995: Markus W. Schäffter: Scheduling with Forbidden Sets

461/1995: Markus W. Schäffter: Drawing Graphs on Rectangular Grids with at most 2 Bends per Edge

458/1995: Ewa Malesińska: List Coloring and Optimization Criteria for a Channel Assignment Problem

447/1995: Martin Henk: Minkowski's second theorem on successive minima 
441/1995: Andreas S. Schulz, Robert Weismantel, Günter M. Ziegler: 0/1-Integer Programming: Optimization and Augmentation are Equivalent, appeared in Paul Spirakis (ed.): Algorithms - ESA '95, Lecture Notes in Computer Science 979, Springer: Berlin, 1995, pp. 473-483

440/1995: Maurice Queyranne, Andreas S. Schulz: Scheduling Unit Jobs with Compatible Release Dates on Parallel Machines with Nonstationary Speeds, appeared in Egon Balas and Jens Clausen (eds.): Integer Programming and Combinatorial Optimization, Lecture Notes in Computer Science 920, Springer: Berlin, 1995, pp. 307-320

439/1995: Rolf H. Möhring, Matthias Müller-Hannemann: Cardinality Matching: Heuristic Search for Augmenting Paths

436/1995: Andreas Parra, Petra Scheffler: Treewidth Equals Bandwidth for AT-Free Claw-Free Graphs

432/1995: Volkmar Welker, Günter M. Ziegler, Rade T. Živaljević: Comparison Lemmas and Applications for Diagrams of Spaces

431/1995: Jürgen Richter-Gebert, Günter M. Ziegler: Realization Spaces of 4-Polytopes are Universal, to appear in Bulletin of the American Mathematical Society, October 1995.

430/1995: Martin Henk: Note on Shortest and Nearest Lattice Vectors

429/1995: Jörg Rambau, Günter M. Ziegler: Projections of Polytopes and the Generalized Baues Conjecture

428/1995: David B. Massey, Rodica Simion, Richard P. Stanley, Dirk Vertigan, Dominic J. A. Welsh, Günter M. Ziegler: Lê Numbers of Arrangements and Matroid Identities

408/1994: Maurice Queyranne, Andreas S. Schulz: Polyhedral Approaches to Machine Scheduling

407/1994: Andreas Parra, Petra Scheffler: How to Use the Minimal Separators of a Graph for Its Chordal Triangulation

401/1994: Rudolf Müller, Andreas S. Schulz: The Interval Order Polytope of a Digraph, appeared in Egon Balas and Jens Clausen (eds.): Integer Programming and Combinatorial Optimization, Lecture Notes in Computer Science 920, Springer: Berlin, 1995, pp. 50-64

396/1994: Petra Scheffler: A Practical Linear Time Algorithm for Disjoint Paths in Graphs with Bounded Tree-width

394/1994: Jens Gustedt: The General Two-Path Problem in time $O(m \log n)$, extended abstract

393/1994: Maurice Queyranne: A Combinatorial Algorithm for Minimizing Symmetric Submodular Functions

392/1994: Andreas Parra: Triangulating Multitolerance Graphs

390/1994: Karsten Weihe: Maximum $(s, t)$-Flows in Planar Networks in $O(|V| \log |V|)$ Time

386/1994: Annelie von Arnim, Andreas S. Schulz: Facets of the Generalized Permutahedron of a Poset, to appear in Discrete Applied Mathematics 
383/1994: Karsten Weihe: Kurzeinführung in C++

377/1994: Rolf H. Möhring, Matthias Müller-Hannemann, Karsten Weihe: Using Network Flows for Surface Modeling

376/1994: Valeska Naumann: Measuring the Distance to SeriesParallelity by Path Expressions

375/1994: Christophe Fiorio, Jens Gustedt: Two Linear Time UnionFind Strategies for Image Processing

374/1994: Karsten Weihe: Edge-Disjoint $(s, t)$-Paths in Undirected Planar graphs in Linear Time

373/1994: Andreas S. Schulz: A Note on the Permutahedron of SeriesParallel Posets, appeared in Discrete Applied Mathematics 57 (1995), pp. $85-90$

371/1994: Heike Ripphausen-Lipa, Dorothea Wagner, Karsten Weihe: Efficient Algorithms for Disjoint Paths in Planar Graphs

Reports may be requested from:

S. Marcus Fachbereich Mathematik, MA 6-1 TU Berlin Straße des 17. Juni 136

D-10623 Berlin - Germany e-mail: Marcus@math.TU-Berlin.DE

Reports are available via anonymous ftp from: ftp.math.tu-berlin.de cd pub/Preprints/combi file Report-<number $>-<$ year $>$.ps.Z

FB Mathematik, MA 6-1, Technische Universität Berlin, Strasse des 17. JUNI 136, D-10623 BERLIN, GERMANY

E-mail address: rambau@math.tu-berlin.de 
